Pacific

Journal of

Mathematics

ON REGULAR HOLONOMIC SYSTEMS WITH SOLUTIONS RAMIFIED ALONG $y^{k}=x^{n}$

Orlando Neto and Pedro C. Silva 


\title{
ON REGULAR HOLONOMIC SYSTEMS WITH SOLUTIONS RAMIFIED ALONG $y^{k}=x^{n}$
}

\author{
Orlando Neto and Pedro C. Silva
}

\begin{abstract}
We classify the holonomic systems of (micro) differential equations of multiplicity one along the conormal of the hypersurface $y^{k}=x^{n}$. We show that their solutions are related to ${ }_{k} F_{k-1}$ hypergeometric functions on the Riemann sphere.
\end{abstract}

\section{Introduction.}

Holonomic $\mathcal{D}$-modules characterize multivalued holomorphic functions in the same way that polynomials characterize algebraic numbers. When we replace systems of differential equations by systems of microdifferential equations we concentrate on the singularities of their multivalued holomorphic solutions modulo holomorphic functions. This point of view was introduced by Riemann with his study of the hypergeometric differential equation and was extended to the several variables case by [19].

The mathematical community isolated a class of multivalued holomorphic functions on the Riemann sphere that arise in many different problems, the special functions. It is an experimental fact that most of the sheaves of solutions of the differential equations that characterize these functions have the remarkable property of being determined by its local monodromies, which can be computed from the indicial equations of these differential equations at their singular points. The local sheaves with the above property are called rigid. We can find in [9] a program of systematic study of special functions based on the concept of rigid local system (see also [22]).

We now have several combinatorial descriptions of germs of regular holonomic $\mathcal{D}$-modules in two variables (see [13], [14], [12]). These remarkable works are not very useful to the specialist in PDE's. He would like to know the equations that define these systems. On the other hand there are too many systems and we cannot expect to obtain the equations for all of them. Pedro C. Silva suggests in [21] a notion of rigidity for germs of holonomic systems of microdifferential equations based on the results of [15], isolating a special class of holonomic systems. The holonomic systems presented in this paper are rigid in the sense of $[\mathbf{2 1}]$. We initiate in this paper a systematic study of special functions on several variables from a microlocal point of view. 
Let $X$ be a complex manifold of dimension $m$. Let $\pi: T^{*} X \rightarrow X$ be the cotangent bundle of $X$. Let $\Lambda$ be a germ of a conic Lagrangian subvariety of $T^{*} X \backslash X$. By Theorem 8.3 of [18], if $\Lambda$ is singular and irreducible and $\Lambda$ is contained in an involutive submanifold of $T^{*} X \backslash X$ of codimension $m-1, \Lambda$ can be identified with the conormal of the hypersurface $y^{k}=x^{n}$. These are the singular Lagrangian varieties with milder singularities. We can find in [18], Theorem 8.6, the classification of systems of microdifferential equations with simple characteristics along $\Lambda$. The purpose of this paper is to classify the systems of microdifferential equations of multiplicity one along $\Lambda$. As a consequence we obtain a classification theorem for $\mathcal{D}$-modules.

Let $k, n$ be integers such that $2 \leq k \leq n-1$ and $(k, n)=1$. Set $\vartheta=$ $x \partial_{x}+\frac{n}{k} y \partial_{y}$. Given complex numbers $\lambda_{i}, i \in \mathbb{Z}$, such that

$$
\lambda_{i+k}=\lambda_{i}, \quad \alpha_{i}:=\lambda_{i}-\lambda_{i+1}+\frac{n-k}{k} \text { is a nonnegative integer, }
$$


and relations

$$
u_{i+k}=u_{i}, \quad\left(\vartheta-\lambda_{i}\right) u_{i}=0, \quad \partial_{x} u_{i}=-\frac{n}{k} x^{\alpha_{i}} \partial_{y} u_{i+1}, \quad \partial_{t_{j}} u_{i}=0,
$$

where $j$ runs over the set $\{1, \ldots, m-2\}$. Notice that

$$
\alpha_{i+k}=\alpha_{i} \quad \text { and } \quad \sum_{i=0}^{k-1} \alpha_{i}=n-k .
$$

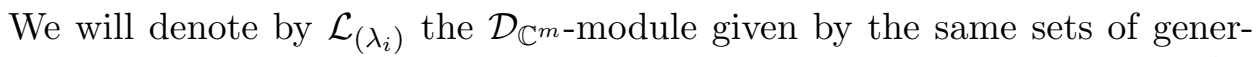
ators and relations. If $2 k+1 \leq n$ and there are $i, j \in \mathbb{Z}$, such that $i \not \equiv j$ $(\bmod k)$ and $\alpha_{i} \alpha_{j} \neq 0$, the system $\mathcal{M}_{\left(\lambda_{i}\right)}$ is not with simple characteristics. These are, as far as the authors know, the first examples of systems of multiplicity one that are not with simple characteristics (see Theorem 2.7 and Proposition 4.5). Moreover, the solutions of the systems $\mathcal{L}_{\left(\lambda_{i}\right)}$ are pullbacks of ${ }_{k} F_{k-1}$ hypergeometric functions on the Riemann sphere twisted by $y^{\lambda \frac{k}{n}}$.

The main results of this paper are stated in Section 4. We study the $\mathcal{O}_{\Lambda}(0)$-module $\mathcal{N} / \mathcal{N}(-1)$, where $\mathcal{N}$ is the canonical lattice of a holonomic system $\mathcal{M}$ with multiplicity one along $\Lambda$. The module $\mathcal{N} / \mathcal{N}(-1)$ gives us a first approximation to the structure of $\mathcal{M}$. We introduce a new invariant, a module over the semigroup of the Legendrian curve $\Lambda / \mathbb{C}^{\times}$, essential to distinguish between systems generated by sections with principal symbols of the same degree of homogeneity. We realized later that the same type of invariant had been used by G.-M. Greuel and G. Pfister (see [4]) in a different context. Theorem 3.5 is the tool that allows the extension of the results on the $\mathcal{O}_{\Lambda}(0)$-module $\mathcal{N} / \mathcal{N}(-1)$ to the $\mathcal{E}_{X}$-module $\mathcal{M}$. In Section 5 we apply the results of Section 4 to $\mathcal{D}$-modules. We obtain a classification theorem for regular holonomic systems with solutions ramified along the 
hypersurface $y^{k}=x^{n}$ verifying some natural conditions and we study their solutions.

We would like to express here our gratitude toward M. Kashiwara for proposing this problem as well as for several useful discussions.

We thank the referee for his remarks.

\section{Systems of microdifferential equations.}

Let $X$ be a complex manifold. Let $\mathcal{D}_{X}$ denote the ring of differential operators on $X$. Let $\mathcal{E}_{X}$ denote the sheaf of microdifferential operators on $X$ (see [19], [20] or [2]). Given $k \in \mathbb{Z}, \mathcal{E}_{X}(k)$ denotes the sheaf of microdifferential operators of order smaller than or equal to $k$. Let $\mathcal{M}$ be an $\mathcal{E}_{X}$-module and $\mathcal{N}$ an $\mathcal{E}_{X}(0)$-submodule of $\mathcal{M}$. Set $\mathcal{N}(k)=\mathcal{E}_{X}(k) \mathcal{N}$.

A coherent $\mathcal{E}_{X^{-}}$-module $\mathcal{M}$ is called a system of microdifferential equations. The support of a system of microdifferential equations is an involutive variety (see [19]). A system of microdifferential equations is called holonomic if its support is Lagrangian.

Let $\mathcal{L}$ be a coherent $\mathcal{O}_{X}$-module and let $Y$ be an irreducible component of the support of $\mathcal{L}$. For each $x \in Y$ set $S_{Y, x}=\left\{f \in \mathcal{O}_{X, x}: f^{-1}(0) \not \supset Y\right\}$. The length of the $S_{Y, x}^{-1} \mathcal{O}_{X, x}$-module $S_{Y, x}^{-1} \mathcal{L}_{x}$ is finite and does not depend on $x$. We call multiplicity of $\mathcal{L}$ along $Y$ to the length of $S_{Y, x}^{-1} \mathcal{L}_{x}$.

Let $\Omega$ be a conic open subset of $T^{*} X \backslash X$. Let $\mathcal{M}$ be a coherent $\left.\mathcal{E}_{X}\right|_{\Omega^{-}}$ module and let $\Lambda$ be an irreducible component of the support of $\mathcal{M}$. Set $I_{\Lambda}=\left\{f \in \mathcal{O}_{\Omega}:\left.f\right|_{\Lambda}=0\right\}$. For each $p \in \Lambda$, there is a coherent $\left.\mathcal{E}_{X}\right|_{U(0)-}$ submodule $\mathcal{N}$ of $\left.\mathcal{M}\right|_{U}$ defined on some conic open neighborhood $U$ of $p$ such that $\left.\mathcal{E}_{X}\right|_{U} \mathcal{N}=\left.\mathcal{M}\right|_{U}$. Set $\overline{\mathcal{M}}=\mathcal{N} / \mathcal{N}(-1)$. The multiplicity along $\Lambda$ of the coherent $\mathcal{O}_{U}$-module

$$
\mathcal{O}_{U} \otimes_{\mathcal{O}_{U}(0)} \overline{\mathcal{M}}
$$

does not depend on $\mathcal{N}$. We call multiplicity of $\mathcal{M}$ along $\Lambda$ to the multiplicity along $\Lambda$ of (4) (see [5]).

Let $\mathcal{L}$ be a coherent $\mathcal{D}_{X}$-module. We call characteristic variety of $\mathcal{L}$ to the support of the coherent $\mathcal{E}_{X}$-module

$$
\mathcal{E}_{X} \otimes_{\pi^{-1} \mathcal{D}_{X}} \pi^{-1} \mathcal{L}
$$

We call multiplicity of $\mathcal{L}$ along $\Lambda$ to the multiplicity of (5) along $\Lambda$.

Set $\mathcal{I}_{\Lambda}=\left\{\left.P \in \mathcal{E}_{X}(1)\right|_{\Omega}: \sigma_{1}(P) \in I_{\Lambda}\right\}$. The morphism $\sigma_{0}: \mathcal{E}_{X}(0) \rightarrow$ $\mathcal{O}_{T^{*} X}(0)$ induces an isomorphism

$$
\left(\left.\mathcal{E}_{X}(0)\right|_{\Omega}\right) / \mathcal{I}_{\Lambda}(-1) \stackrel{\sim}{\longrightarrow} \mathcal{O}_{\Lambda}(0) .
$$

Proposition 2.1. If $\mathcal{M}$ is a holonomic $\left.\mathcal{E}_{X}\right|_{\Omega}$-module and $\mathcal{M}^{\prime}$ is a coherent $\left.\mathcal{E}_{X}\right|_{\Omega^{-}}$submodule of $\mathcal{M}$ such that mult $_{\Lambda} \mathcal{M}=$ mult $_{\Lambda} \mathcal{M}^{\prime}$ for each irreducible component of the support of $\mathcal{M}, \mathcal{M}=\mathcal{M}^{\prime}$. 
Proof. This is an immediate consequence of the fact that all irreducible components of the support of a coherent $\left.\mathcal{E}_{X}\right|_{\Omega}$-module have dimension greater than or equal to $\operatorname{dim} X$.

Definition 2.2. Let $\Omega$ be a conic open subset of $T^{*} X \backslash X$. Let $\Lambda$ be a conic Lagrangian subvariety of $\Omega$. Let $\mathcal{M}$ be coherent $\left.\mathcal{E}_{X}\right|_{\Omega}$-module with support $\Lambda$. We say that $\mathcal{M}$ is regular holonomic at a point $a \in \Lambda$ if there is a conic open neighborhood $U$ of $a$ and a coherent $\left.\mathcal{E}_{X}(0)\right|_{U}$-submodule $\mathcal{N}$ of $\left.\mathcal{M}\right|_{U}$ such that $\left.\mathcal{E}_{X}\right|_{U} \mathcal{N}=\left.\mathcal{M}\right|_{U}$ and $\left.\mathcal{I}_{\Lambda}\right|_{U} \mathcal{N}=\mathcal{N}$. We say that $\mathcal{M}$ is regular holonomic if the set of points $a \in \Lambda$ such that $\mathcal{M}$ is not regular at $a$ is nowhere dense in $\Lambda$.

The following result was obtained taking $c=0$ in Theorem 5.1.6 of [7].

Theorem 2.3. Let $\mathcal{M}$ be a regular holonomic system of microdifferential equations with support $\Lambda \subset T^{*} X \backslash X$. Then $\mathcal{M}$ has a canonical coherent



1) $\mathcal{E}_{X} \mathcal{N}=\mathcal{M}$ and $\mathcal{I}_{\Lambda} \mathcal{N}=\mathcal{N}$.

2) The support of a section of the sheaf $\mathcal{M} / \mathcal{N}$ is an analytic set of codimension smaller than or equal to the dimension of $X$.

We call $\mathcal{N}$ the canonical lattice of $\mathcal{M}$.

Given a left ideal $\mathcal{I}$ of $\mathcal{E}_{X}$, let $\overline{\mathcal{I}}$ be the ideal of $\mathcal{O}_{T^{*} X}$ generated by the principal symbols of the microdifferential operators $P \in \mathcal{I}$. We call $\overline{\mathcal{I}}$ the ideal of symbols of the ideal $\mathcal{I}$.

Definition 2.4. Let $\mathcal{M}$ be a holonomic system of microdifferential equations with support $\Lambda$. Let $U$ be a conic open neighborhood of $a \in \Lambda$. Let $u$ be a generator of $\left.\mathcal{M}\right|_{U}$. Let $\mathcal{I}$ denote the annihilator of $u$. The section $u$ is called a local generator of $\mathcal{M}$ with simple characteristics if $\overline{\mathcal{I}}$ equals the defining ideal of $\Lambda \cap U$. The module $\mathcal{M}$ has simple characteristics along $\Lambda$ if it admits a generator with simple characteristics in a neighborhood of each point of $\Lambda$.

It follows from Theorem 2.5 that a holonomic system $\mathcal{M}$ has multiplicity one along a Lagrangian variety $\Lambda$ if and only if $\mathcal{M}$ has simple characteristics along the regular part of $\Lambda$.

Theorem 2.5. Let $\Omega$ be an open subset of $T^{*} X \backslash X$. Let $\mathcal{M}$ be a holonomic $\left.\mathcal{E}_{X}\right|_{\Omega}$-module with support $\Lambda$. The following holds.

1) If $\mathcal{M}$ has simple characteristics along $\Lambda$ then $\mathcal{M}$ is regular holonomic with multiplicity one along the irreducible components of $\Lambda$.

2) If $\mathcal{M}$ has multiplicity one along the irreducible components of $\Lambda$ then $\mathcal{M}$ has simple characteristics along $\Lambda \backslash \operatorname{Sing}(\Lambda)$. Moreover, $\mathcal{M}$ is regular holonomic. 
Proof. The first statement follows from Theorem I. 6.3.2 of [20].

Given $q \in \Lambda$ there is a conic open neighborhood $U$ of $q$ and a section $u$ of $\left.\mathcal{M}\right|_{U}$ that does not vanish on $\Lambda \cap U$. Since $\operatorname{mult}_{\Lambda \cap U}\left(\mathcal{E}_{X} u\right) \geq 1$ and $\left.\mathcal{E}_{X} u \subset \mathcal{M}\right|_{U}, \mathcal{E}_{X} u=\left.\mathcal{M}\right|_{U}$ by Proposition 2.1. Set $\overline{\mathcal{M}}=\mathcal{E}_{X}(0) u / \mathcal{E}_{X}(-1) u$. Since

$$
\operatorname{mult}_{\Lambda} \mathcal{M}=\sum_{k \geq 0} \operatorname{mult}_{\Lambda}\left(I_{\Lambda}^{k} \otimes \overline{\mathcal{M}} / I_{\Lambda}^{k+1} \otimes \overline{\mathcal{M}}\right)
$$

(see [20], Appendix D) and $I_{\Lambda}^{k} \otimes \overline{\mathcal{M}}=I_{\Lambda}^{k+1} \otimes \overline{\mathcal{M}} \Rightarrow I_{\Lambda}^{k+1} \otimes \overline{\mathcal{M}}=I_{\Lambda}^{k+2} \otimes \overline{\mathcal{M}}$, $\operatorname{mult}_{\Lambda}\left(\mathcal{O}_{T^{*} X} \otimes \overline{\mathcal{M}} / I_{\Lambda} \otimes \overline{\mathcal{M}}\right)=1$ and there is a dense Zariski open subset $U_{0}$ of $U$ such that $\left.I_{\Lambda} \otimes \overline{\mathcal{M}}\right|_{U_{0}}=\left.I_{\Lambda}^{2} \otimes \overline{\mathcal{M}}\right|_{U_{0}}$. Hence $\left.I_{\Lambda} \otimes \overline{\mathcal{M}}\right|_{U_{0}}=\left.\cap_{k \geq 1} I_{\Lambda}^{k} \otimes \overline{\mathcal{M}}\right|_{U_{0}}=$ 0 . Let $q_{0} \in U_{0}$ be a nonsingular point of $\Lambda$. We can assume that $q_{0}=\left(0, d x_{1}\right)$ and $\Lambda=\left\{x_{1}=\xi_{2}=\cdots=\xi_{n}=0\right\}$. There are $R_{1}, \ldots, R_{n} \in \mathcal{E}_{X, q_{0}}(-1)$ such that

$$
\left(x_{1}-R_{1}\right) u_{q_{0}}=\left(\partial_{x_{2}} \partial_{x_{1}}^{-1}-R_{2}\right) u_{q_{0}}=\cdots=\left(\partial_{x_{n}} \partial_{x_{1}}^{-1}-R_{n}\right) u_{q_{0}}=0 .
$$

Hence the ideal of symbols of the annihilator of $u_{q_{0}}$ equals $I_{\Lambda, q_{0}}$. We have shown in this way that $\mathcal{M}$ has simple characteristics at a generic point of $\Lambda$, hence is regular holonomic. By the classification theorem for regular holonomic $\mathcal{E}_{X}$-modules with smooth support (see for instance Remark 6.7 of $[8]), \mathcal{M}$ has simple characteristics along $\Lambda \backslash \operatorname{Sing}(\Lambda)$.

The following result is a consequence of Theorem 5.1.6 of [7] and Theorem I.6.3.3 of $[\mathbf{2 0}]$.

Corollary 2.6. Let $\mathcal{M}$ be a holonomic systems of microdifferential equations with simple characteristics along $\Lambda$. Let $\mathcal{N}$ be the canonical lattice of $M$. Given $a \in \Lambda$ there is a conic open neighborhood $U$ of a and a section $u$ of $\mathcal{M}$ on $U$ such that $u$ is a generator of $\left.\mathcal{M}\right|_{U}$ with simple characteristics along $\Lambda$ and $\left.\mathcal{N}\right|_{U}=\mathcal{E}_{X}(0) u$.

Theorem 2.7. Let $\Lambda$ be the germ at a point $p$ of an irreducible Lagrangian variety of $T^{*} X \backslash X$. Let $M$ be the fiber at $p$ of a regular holonomic $\mathcal{E}_{X-}$ module. Let $N$ be the canonical lattice of $M$.

1) The module $M$ has multiplicity one along $\Lambda$ if and only if $N / N(-1)$ is a finitely generated torsion free $\mathcal{O}_{\Lambda, p}(0)$-module of rank one.

2) The module $M$ has simple characteristics along $\Lambda$ if and only if $N / N(-1)$ is a free $\mathcal{O}_{\Lambda, p}(0)$-module of dimension one.

Proof. Since $N$ is a coherent $\mathcal{E}_{X, p}$-module, $N / N(-1)$ is finitely generated. By Theorem 2.5, the support of $I_{\Lambda, p}(0)(N / N(-1))$ is contained in the singular locus of $\Lambda$. By Theorem 2.3, $I_{\Lambda, p}(0)(N / N(-1))=0$. Hence $N / N(-1)$ is an $\mathcal{O}_{\Lambda, p}(0)$-module. By Theorem 2.3 $N / N(-1)$ is a torsion free $\mathcal{O}_{\Lambda, p}(0)$-module. 
Set $S_{\Lambda, p}(0)=S_{\Lambda, p} \cap \mathcal{O}_{T^{*} X, p}(0)$. The $\mathcal{E}_{X, p^{-m o d u l e}} M$ has multiplicity one along $\Lambda$ if and only if

$$
S_{\Lambda, p}^{-1}\left(\mathcal{O}_{T^{*} X, p} \otimes_{\mathcal{O}_{T^{*} X, p}(0)} N / N(-1)\right)
$$

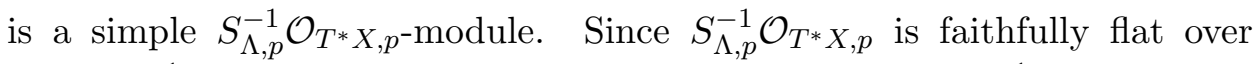
$\left(S_{\Lambda, p}(0)\right)^{-1} \mathcal{O}_{T^{*} X, p}(0),(7)$ is simple if and only if $\left(S_{\Lambda, p}(0)\right)^{-1}(N / N(-1))$ is a simple $\left(S_{\Lambda, p}(0)\right)^{-1} \mathcal{O}_{T^{*} X, p}(0)$-module. Since $\left(S_{\Lambda, p}(0)\right)^{-1} \mathcal{O}_{\Lambda, p}(0)$ is the quotient field of $\mathcal{O}_{\Lambda, p}(0), M$ has multiplicity one along $\Lambda$ if and only if $N / N(-1)$ has rank one.

Assume that $\mathcal{M}$ has simple characteristics along $\Lambda$. By Corollary 2.6 there is a local generator $u$ of $\mathcal{M}$, with simple characteristics along $\Lambda$, such that $N=\mathcal{E}_{X, p}(0) u$. Hence $N / N(-1)$ is a torsion free $\mathcal{O}_{\Lambda, p}(0)$-module generated by $u+N(-1)$.

Assume that $N / N(-1)$ is a free $\mathcal{O}_{\Lambda, p}(0)$-module generated by a section $v$. Let $u$ be a section of $N$ such that $v=u+N(-1)$. Since $N=\mathcal{E}_{X}(0) u+\mathcal{E}_{X}(-k) N$ for all $k \geq 0$, it follows from a theorem of [20] that $N=\mathcal{E}_{X}(0) u$. Let $f \in I_{\Lambda, p}(0)$. Since $f v=0$, there is $Q \in \mathcal{E}_{X, p}(0)$ such that $\sigma(Q)=f$ and $Q u \in \mathcal{E}_{X, p}(-1) u$. Hence there is $P \in \mathcal{E}_{X, p}(0)$ such that $\sigma(P)=f$ and $P u=0$. Hence $u$ is a generator with simple characteristics along $\Lambda$.

\section{A microdifferential Cauchy problem.}

Throughout this section we will assume $m=2$. We shall denote by $\mathbb{N}$ the set of nonnegative integers.

Let $X$ be an open set of $\mathbb{C}^{2}$ containing the origin. Let $(x, y)$ be a system of coordinates of $\mathbb{C}^{2}$. Let $(x, y, \xi, \eta)$ be the associated system of coordinates of $T^{*} \mathbb{C}^{2}$ such that $\omega=\xi d x+\eta d y$ is the canonical one-form.

Let $d$ be a positive integer. Given $f \in \mathrm{M}_{d}\left(\mathcal{O}_{T^{*} X}(-j)\right), g \in \mathrm{M}_{d}\left(\mathcal{O}_{T^{*} X}(-i)\right)$, $i, j, \alpha, \beta \in \mathbb{N}$, we set (see [17]),

$$
(f, g)^{(\alpha, \beta)}=\frac{1}{\alpha ! \beta !} \frac{\partial^{\alpha+\beta} f}{\partial \xi^{\alpha} \partial \eta^{\beta}} \frac{\partial^{\alpha+\beta} g}{\partial x^{\alpha} \partial y^{\beta}} .
$$

Let $P, Q \in \mathrm{M}_{d}\left(\mathcal{E}_{X}(0)(\Omega)\right)$ for some open neighborhood $\Omega$ of $(0, d y)$ such that $P=\sum_{l \geq 0} p_{l} \eta^{-l}$ and $Q=\sum_{l \geq 0} q_{l} \eta^{-l}$ with $p_{l}, q_{l} \in \mathrm{M}_{d}(\mathbb{C}\{x, y, p\})$. The product of the matrices $P, Q$ is given by the Leibniz rule

$$
P Q=\sum_{l \geq 0} \sum_{\mu+\nu+\alpha+\beta=l}\left(p_{\mu} \eta^{-\mu}, q_{\nu} \eta^{-\nu}\right)^{(\alpha, \beta)} .
$$

We will consider in $\Omega$ the coordinate system $\left(x_{0}, y_{0}, p_{0}, \zeta\right)$, where $x_{0}=-x$, $y_{0}=-y, p_{0}=-\xi / \eta$ and $\zeta=\eta$. Remark that $\partial_{x}=-\partial_{x_{0}}, \partial_{y}=-\partial_{y_{0}}$, $\partial_{\xi}=-\zeta^{-1} \partial_{p_{0}}$ and $\partial_{\eta}=\partial_{\zeta}-\zeta^{-1} p_{0} \partial_{p_{0}}$. Hence, $x \partial_{x}=x_{0} \partial_{x_{0}}, y \partial_{y}=y_{0} \partial_{y_{0}}$, 
$\xi \partial_{\xi}=p_{0} \partial_{p_{0}}$ and $\eta \partial_{\eta}=\zeta \partial_{\zeta}-p_{0} \partial_{p_{0}}$. Given $\varphi, \psi \in \mathbb{C}\left\{x_{0}, y_{0}, p_{0}\right\}$,

$$
\begin{aligned}
& \partial_{\xi}^{\alpha}\left(\varphi \zeta^{-\mu}\right)=(-1)^{\alpha}\left(\partial_{p_{0}}^{\alpha} \varphi\right) \zeta^{-\mu-\alpha} \\
& \partial_{\eta}^{\beta}\left(\varphi \zeta^{-\mu}\right)=(-1)^{\beta}\left(\prod_{0 \leq k<\beta}\left(\mu+k+p_{0} \partial_{p_{0}}\right) \varphi\right) \zeta^{-\mu-\beta}, \\
& \partial_{x}^{\alpha}\left(\psi \zeta^{-\nu}\right)=(-1)^{\alpha}\left(\partial_{x_{0}}^{\alpha} \psi\right) \zeta^{-\nu}, \\
& \partial_{y}^{\beta}\left(\psi \zeta^{-\nu}\right)=(-1)^{\beta}\left(\partial_{y_{0}}^{\beta} \psi\right) \zeta^{-\nu} .
\end{aligned}
$$

Moreover, $\left(\varphi \zeta^{-\mu}, \psi \zeta^{-\nu}\right)^{(\alpha, \beta)}$ equals

$$
\frac{1}{\alpha ! \beta !}\left(\partial_{p_{0}}^{\alpha} \prod_{0 \leq k<\beta}\left(\mu+k+p_{0} \partial_{p_{0}}\right) \varphi\right)\left(\partial_{x_{0}}^{\alpha} \partial_{y_{0}}^{\beta} \psi\right) \zeta^{-\mu-\nu-\alpha-\beta} .
$$

A formal expression

$$
U=\sum_{l \geq 0} u_{l} \zeta^{-l}, \quad u_{l} \in \mathbb{C}\left\{x_{0}, y_{0}, p_{0}\right\},
$$

defines a microdifferential operator near $q=(0, d y)$ if and only if there exists an open neighborhood $\Gamma$ of $(0,0,0)$ where the $u_{l}$ 's converge and

$$
\limsup _{l \rightarrow \infty} \sqrt[l]{\sup _{z \in \Gamma}\left|u_{l}(z)\right| / l !}<\infty .
$$

Given formal expressions $\bar{U}=\sum_{l \geq 0} \bar{u}_{l} \zeta^{-l}$ and $U=\sum_{l \geq 0} u_{l} \zeta^{-l}$ where $\bar{u}_{l}, u_{l} \in$ $\mathbb{C}\left\{x_{0}, y_{0}, p_{0}\right\}$ we denote $\bar{U} \gg U$ if there is $N \in \mathbb{Z}, K \in \mathbb{R}, N, K>0$, such that $K^{l} \bar{u}_{l} \gg u_{l}$ (i.e., $K^{l} \bar{u}_{l}$ estimates $u_{l}$ ) for all $l \geq N$. In view of (10) if $\bar{U} \gg U$ and $\bar{U}$ defines a microdifferential operator near $(0, d y), U$ also defines a microdifferential operator near $(0, d y)$. We extend the notation to matrices in the obvious way. In order to prove that a formal expression as above defines a convergent microdifferential operator, we introduce a microlocal version of the majorant method. The following lemma follows immediately from (9):

Lemma 3.1. Let $\varphi_{1}, \bar{\varphi}_{1}, \varphi_{2}, \bar{\varphi}_{2} \in M_{d}\left(\mathbb{C}\left\{x_{0}, y_{0}, p_{0}\right\}\right)$ and let $\alpha, \beta, \mu, \nu \in \mathbb{N}$. Set $\bar{\varphi} \zeta^{-\mu-\nu-\alpha-\beta}=\left(\bar{\varphi}_{1} \zeta^{-\mu}, \bar{\varphi}_{2} \zeta^{-\nu}\right)^{(\alpha, \beta)}, \varphi \zeta^{-\mu-\nu-\alpha-\beta}=\left(\varphi_{1} \zeta^{-\mu}, \varphi_{2} \zeta^{-\nu}\right)^{(\alpha, \beta)}$ with $\bar{\varphi}, \varphi \in M_{d}\left(\mathbb{C}\left\{x_{0}, y_{0}, p_{0}\right\}\right)$. If $\bar{\varphi}_{1} \gg \varphi_{1}$ and $\bar{\varphi}_{2} \gg \varphi_{2}$ then $\bar{\varphi} \gg \varphi$.

The two following results are particular cases of the main result in [16] and its proof.

Lemma 3.2. Let $c_{1}, \ldots, c_{t}$ be complex numbers such that 0 does not belong to its convex hull. Let $b, f \in \mathbb{C}\left\{z_{1}, \ldots, z_{t}\right\}$ be analytic functions at the origin. 
Assume that $b(0) \notin c_{1} \mathbb{N}+\cdots+c_{t} \mathbb{N}$ or $b(0)=0$. There is $u \in \mathbb{C}\left\{z_{1}, \ldots, z_{t}\right\}$ solution of

$$
\left(c_{1} z_{1} \frac{\partial}{\partial z_{1}}+\cdots+c_{t} z_{t} \frac{\partial}{\partial z_{t}}-b\right) u=f
$$

if and only if $f \in\left(z_{1}, \ldots, z_{t}, b\right)$. In the former case the solution $u$ is unique. In the later case there is a one to one correspondence between the solutions of (11) and the Cauchy data $u(0)$.

Lemma 3.3. Let $c_{1}, \ldots, c_{t}$ be complex numbers such that 0 does not belong to its convex hull and $\lambda^{\prime}$ a complex number such that $\left\{\lambda^{\prime}\right\} \cap\left(c_{1} \mathbb{N}+\cdots+c_{t} \mathbb{N}\right) \subset$ $\{0\}$. Then there exists an $\varepsilon>0$ verifying,

$$
\left|l_{1} c_{1}+\cdots+l_{t} c_{t}-\lambda^{\prime}\right| \geq \varepsilon\left(l_{1}+\cdots+l_{t}\right), \quad \forall l_{1}, \ldots, l_{t} \in \mathbb{N} .
$$

Given $a, b \in \mathbb{R}, 0<a<b$, and $c \in(\mathbb{C} \backslash \mathbb{R}) \cup] 0,1\left[\right.$, set $P=c x_{0} \partial_{x_{0}}+y_{0} \partial_{y_{0}}+$ $(1-c) p_{0} \partial_{p_{0}}$ and $\bar{P}=a x_{0} \partial_{x_{0}}+b y_{0} \partial_{y_{0}}+(b-a) p_{0} \partial_{p_{0}}$.

Lemma 3.4. Given $\lambda^{\prime} \in \mathbb{C}$ such that $\left\{\lambda^{\prime}\right\} \cap(c \mathbb{N}+(1-c) \mathbb{N}) \subset\{0\}$ there exists $\delta=\delta\left(a, b, c, \lambda^{\prime}\right)>0$, such that for $l \in \mathbb{N}, l \geq 1, \varphi, \bar{\varphi} \in \mathbb{C}\left\{x_{0}, y_{0}, p_{0}\right\}$,

$$
(\bar{P}+b l) \bar{\varphi} \gg\left(P+l-\lambda^{\prime}\right) \varphi \Rightarrow \bar{\varphi} \gg \delta \varphi .
$$

Proof. Write

$$
\varphi=\sum_{\alpha, \beta, \gamma \geq 0} \varphi_{\alpha, \beta, \gamma} x_{0}{ }^{\alpha} y_{0}{ }^{\beta} p_{0}{ }^{\gamma}, \quad \bar{\varphi}=\sum_{\alpha, \beta, \gamma \geq 0} \bar{\varphi}_{\alpha, \beta, \gamma} x_{0}{ }^{\alpha} y_{0}{ }^{\beta} p_{0}{ }^{\gamma} .
$$

The left-hand side of (12) is equivalent to say that

$$
((\alpha+\beta+l) a+(\beta+\gamma+l)(b-a)) \bar{\varphi}_{\alpha, \beta, \gamma}
$$

estimates

$$
\left|(\alpha+\beta+l) c+(\beta+\gamma+l)(1-c)-\lambda^{\prime}\right|\left|\varphi_{\alpha, \beta, \gamma}\right|,
$$

for all $\alpha, \beta, \gamma \geq 0$ and for all $l \geq 1$. By the hypothesis on $c$ and $\lambda^{\prime}$ along with Lemma 3.3, there is an $\varepsilon>0$ such that for all $\alpha, \beta, \gamma \geq 0$ and for all $l \geq 1$

$$
\left|(\alpha+\beta+l) c+(\beta+\gamma+l)(1-c)-\lambda^{\prime}\right| \geq \varepsilon((\alpha+\beta+l)+(\beta+\gamma+l)) .
$$

Choosing $\delta \in \mathbb{R}, 0<\delta<\varepsilon / \max \{a, b-a\}$,

$$
\left|(\alpha+\beta+l) c+(\beta+\gamma+l)(1-c)-\lambda^{\prime}\right|
$$

estimates

$$
\delta((\alpha+\beta+l) a+(\beta+\gamma+l)(b-a))>0,
$$

which implies the right-hand side of (12).

In the sequel we shall denote the identity matrix of order $t$ by $I_{t}$. 
Theorem 3.5. Consider the microdifferential Cauchy problem

$$
\left\{\begin{array}{l}
{\left[\vartheta^{\prime}, U\right]-\left[A_{0}, U\right]-\mathcal{A}_{-1} U=0} \\
\sigma_{0}(U)((0, d y))=I_{d}
\end{array}\right.
$$

where $\left.\vartheta^{\prime}=c x \partial_{x}+y \partial_{y}, c \in(\mathbb{C} \backslash \mathbb{R}) \cup\right] 0,1\left[, A_{0} \in M_{d}(\mathbb{C})\right.$ and $\mathcal{A}_{-1} \in$ $M_{d}\left(\mathcal{E}_{X(-1)}\right)$. Assume that $A_{0}$ is semisimple with eigenvalues $\lambda_{0}^{\prime}, \ldots, \lambda_{d-1}^{\prime}$ verifying

$$
\left\{\lambda_{i}^{\prime}-\lambda_{j}^{\prime}: 0 \leq i, j \leq d-1\right\} \cap(c \mathbb{N}+(1-c) \mathbb{N}) \subset\{0\} .
$$

Then there exists, in a neighborhood $\Omega$ of $(0, d y)$, one and only one invertible matrix $U \in M_{d}\left(\mathcal{E}_{X}(0)\right)(\Omega)$ solution of $(13)$.

Proof. We can assume that $A_{0}=\operatorname{diag}\left(\lambda_{0}^{\prime}, \ldots, \lambda_{d-1}^{\prime}\right)$ and $\mathcal{A}_{-1}=\sum_{l \geq 1} a_{l} \zeta^{-l}$ with $a_{l} \in \mathbb{C}\left\{x_{0}, y_{0}, p_{0}\right\}$ for all $l$.

Let $f=\sigma\left(\vartheta^{\prime}\right)=c x \xi+y \zeta$ be the principal symbol of $\vartheta^{\prime}$ and let $H_{f}=$ $c x \partial_{x}+y \partial_{y}-c p \partial_{p}-\eta \partial_{\eta}=c x_{0} \partial_{x_{0}}+y_{0} \partial_{y_{0}}+(1-c) p_{0} \partial_{p_{0}}-\zeta \partial_{\zeta}=P-\zeta \partial_{\zeta}$ be the corresponding Hamiltonian vector field. Let us find a formal series

$$
U=\sum_{l \geq 0} u_{l} \zeta^{-l}, \quad u_{l} \in \mathrm{M}_{d}\left(\mathbb{C}\left\{x_{0}, y_{0}, p_{0}\right\}\right),
$$

such that $H_{f}\left(u_{l} \zeta^{-l}\right)-\left[A_{0}, u_{l}\right] \zeta^{-l}=\omega_{l}, \forall l \geq 0$, where

$$
\omega_{l}=\sum_{\substack{\nu+\mu+\alpha+\beta=l \\ \mu \geq 1}}\left(a_{\mu} \zeta^{-\mu}, u_{\nu} \zeta^{-\nu}\right)^{(\alpha, \beta)}, \quad \alpha, \beta, \nu, \mu \in \mathbb{N} .
$$

The matrices $u_{j}$ are solution of the system of differential equations

$$
(P+l) u_{l}-\left[A_{0}, u_{l}\right]=\omega_{l}, \quad l \geq 0 .
$$

Since $\omega_{0}=0, u_{0}=I_{d}$ is the solution of (16) for $l=0$ with Cauchy data $u_{0}(0)=I_{d}$ (see Lemma 3.2). Assume that $l \geq 1$. Set $\lambda_{i, j}^{\prime}=\lambda_{i}^{\prime}-\lambda_{j}^{\prime}$, $i, j=0, \ldots, d-1$. Writing $u_{l}=\left(u_{l ; i, j}\right)_{i, j}$ and $\omega_{l}=\left(\omega_{l ; i, j}\right)_{i, j}$, the system $(16)$ is equivalent to the system of $d^{2}$ first order linear differential equations with degenerate principal symbols

$$
\left(P+l-\lambda_{i, j}^{\prime}\right) u_{l ; i, j}=\omega_{l ; i, j}, \quad i, j \in\{0, \ldots, d-1\} .
$$

It follows from (15) that $\omega_{l ; i, j}$ only depends on the entries $u_{\nu ; i, j}$ for $\nu<l$.

By the hypothesis zero does not belong to the convex hull of $\{c, 1-c, 1\}$. Since $l \geq 1, \lambda_{i, j}^{\prime}-l \neq 0$. By Lemma 3.2 we can find, recursively, unique analytic functions $u_{l ; i, j}, i, j=0, \ldots, d-1$, verifying (17). In order to finish the proof of Theorem 3.5 it is enough to show that $U=\sum_{l \geq 0} u_{l ; i, j} \zeta^{-l}$, $i, j \in\{0, \ldots, d-1\}$, is the symbol of a convergent microdifferential operator.

We denote by $(e)_{d}$ the matrix of type $d \times d$ with all the entries equal to $e$. Notice that $(e)_{d}\left(e^{\prime}\right)_{d}=d\left(e e^{\prime}\right)_{d}$. There is a convergent microdifferential operator $Q=\sum_{l \geq 1} q_{l} \zeta^{-l}$, defined in a small neighborhood of $(0, d y)$, such 
that $\left(q_{l}\right)_{d} \gg a_{l}$ for all $l \geq 1$ (see for instance the proof of Theorem 3.2 of $[\mathbf{1 7}])$. Set $\bar{\vartheta}=a x \partial_{x}+b y \partial_{y}$ where $a, b \in \mathbb{R}, 0<a<b$. Set $\bar{f}:=\sigma(\bar{\vartheta})=a x \xi+$ by . Then $H_{\bar{f}}=\bar{P}-b \zeta \partial_{\zeta}$, where $\bar{P}=a x_{0} \partial_{x_{0}}+b x_{0} \partial_{y_{0}}+(b-a) p_{0} \partial_{p_{0}}$. Let $V=\sum_{l \geq 0} v_{l} \zeta^{-l} \in \mathcal{E}_{X}(0)$ be the unique invertible microdifferential operator such that

$$
V^{-1}(\bar{\vartheta}-Q) V=\bar{\vartheta}, \quad \sigma_{0}(V)(0, d y)=1
$$

(see Lemma 3.2 in $[\mathbf{1 7}]$ ). Actually $v_{0}=1$ is the unique solution of the Cauchy problem with degenerate principal symbols, $\bar{P} v_{0}=0, v_{0}(0)=1$, and $v_{l}, l \geq 1$, is determined recursively by $(\bar{P}+b l) v_{l}=\bar{\omega}_{l}$ where

$$
\bar{\omega}_{l} \zeta^{-l}=\sum_{\substack{\nu+\mu+\alpha+\beta=l \\ \mu \geq 1}}\left(q_{\mu} \zeta^{-\mu}, v_{\nu} \zeta^{-\nu}\right)^{(\alpha, \beta)}
$$

Fix $\delta \in \mathbb{R}, 0<\delta \ll 1$ in conditions of Lemma 3.4 for all $\lambda_{i, j}^{\prime}$ 's. Set $\varepsilon=\delta^{-1} d$. Let us prove by induction that $\varepsilon^{l}\left(v_{l}\right)_{d} \gg u_{l}$ for all $l$, that is, $(V)_{d} \gg U$. By the hypothesis, $\left(v_{0}\right)_{d}=(1)_{d} \gg u_{0}=I_{d}$. Assume that $\varepsilon^{\nu}\left(v_{\nu}\right)_{d} \gg u_{\nu}$ for $\nu \leq l-1$. Since $\left(q_{\mu}\right)_{d} \gg a_{\mu}$, Lemma 3.1 yields

$$
\sum_{\substack{\nu+\mu+\alpha+\beta=l \\ \mu \geq 1}}\left(\left(q_{\mu} \zeta^{-\mu}\right)_{d}, \varepsilon^{\nu}\left(v_{\nu} \zeta^{-\nu}\right)_{d}\right)^{(\alpha, \beta)} \gg \sum_{\substack{\nu+\mu+\alpha+\beta=l \\ \mu \geq 1}}\left(a_{\mu} \zeta^{-\mu}, u_{\nu} \zeta^{-\nu}\right)^{(\alpha, \beta)} .
$$

Since $\varepsilon \geq 1$, the left-hand side of the previous relation is estimated by

$$
\sum_{\substack{\nu+\mu+\alpha+\beta=l \\ \mu \geq 1}}\left(\left(q_{\mu} \zeta^{-\mu}\right)_{d}, \varepsilon^{l-1}\left(v_{\nu} \zeta^{-\nu}\right)_{d}\right)^{(\alpha, \beta)}
$$

that equals

$$
d \varepsilon^{l-1}\left(\sum_{\substack{\nu+\mu+\alpha+\beta=l \\ \mu \geq 1}}\left(q_{\mu} \zeta^{-\mu}, v_{\nu} \zeta^{-\nu}\right)^{(\alpha, \beta)}\right)_{d} .
$$

Therefore by definition of $\omega_{l}$ and $\bar{\omega}_{l}$ we have $d \varepsilon^{l-1} \bar{\omega}_{l} \gg \omega_{l ; i, j}$ for all $i, j$ hence

$$
d \varepsilon^{l-1}(\bar{P}+b l) v_{l} \gg\left(P+l-\lambda_{i, j}^{\prime}\right) u_{l ; i, j},
$$

for all $i, j \in\{0, \ldots, d-1\}$. By Lemma $3.4, \varepsilon^{l} v_{l} \gg u_{l ; i, j}$ for all $i, j$.

\section{Main results.}

In the following we shall denote the nilpotent Jordan block of order $t$ by $N_{t}$.

Lemma 4.1. Let $\alpha \in \mathbb{C}$ and assume that $Y \in M_{\mu \times \nu}\left(\mathbb{C}\{x\}\left[x^{-1}\right]\right)$ verifies the differential equation

$$
\left(x \frac{d}{d x}-\alpha\right) Y=Y N_{\nu}-N_{\mu} Y
$$


Then $Y=C x^{\alpha}$, where $C \in M_{\mu \times \nu}(\mathbb{C})$ verifies $C N_{\nu}-N_{\mu} C=0$. In particular, if $\alpha \notin \mathbb{Z}, Y=0$.

Proof. We will identify a matrix $Y=\left(a_{i, j}\right) \in \mathrm{M}_{\mu \times \nu}$ with a family $\left(a_{i, j}\right)$, $i, j \in \mathbb{Z}$, such that $a_{i, j}=0$ if $i \notin\{0, \ldots, \mu-1\}$ or $j \notin\{0, \ldots, \nu-1\}$. We will show by recursion on $t$ that there is a constant $c_{t}$ such that $a_{i, i+t}=c_{t} x^{\alpha}$ if $0 \leq i \leq \mu-1,0 \leq i+t \leq \nu-1$. The statement is true for $t$ small enough. Let us assume that it holds for a certain $t$. Then

$$
\left(x \frac{d}{d x}-\alpha\right) a_{i, i+t+1}=a_{i, i+t}-a_{i+1, i+t+1}=0 .
$$

Hence there are complex numbers $c_{i, t+1}$ such that $a_{i, i+t+1}=c_{i, t+1} x^{\alpha}$. Moreover,

$$
\left(x \frac{d}{d x}-\alpha\right) a_{i, i+t+2}=\left(c_{i, t+1}-c_{i+1, t+1}\right) x^{\alpha} .
$$

Hence $c_{i, t+1}$ does not depend on $i$.

Theorem 4.2. Let $L$ be a free $\mathbb{C}\{x\}$-module of dimension $k$. Let $\nabla$ be a $\mathbb{C}$-linear endomorphism of $L$ such that

$$
\nabla(f u)=x \frac{d f}{d x} u+f \nabla u, f \in \mathbb{C}\{x\}, u \in L .
$$

Let $p$ be a $\mathbb{C}\{x\}$-linear endomorphism of $L$ such that $[\nabla, p]=((n-k) / k) p$ and $p^{k}=(n / k)^{k} x^{n-k}$. There are complex numbers $\lambda_{i}, i \in \mathbb{Z}$, and a system of generators of $L, u_{i}, i \in \mathbb{Z}$, such that (1) holds, $\left(u_{0}, \ldots, u_{k-1}\right)$ is a basis of $L$ and

$$
u_{i+k}=u_{i}, \quad \nabla u_{i}=\lambda_{i} u_{i}, \quad p u_{i}=\frac{n}{k} x^{\alpha_{i}} u_{i+1}, \quad i \in \mathbb{Z} .
$$

Proof. Let $A=\left(a_{i, j}\right)$ and $B=\left(b_{i, j}\right)$ be respectively the matrices of the actions of $\nabla$ and $p$ with respect to a basis $\left(u_{0}, \ldots, u_{k-1}\right)$ of $L$. Set $u_{j}=u_{i}$ if $j-i \equiv 0(\bmod k)$, for each $j \in \mathbb{Z}$. Since $\nabla\left(p u_{i}\right)=[\nabla, p] u_{i}+p \nabla u_{i}$,

$$
\sum_{j=0}^{k-1} x \frac{d b_{j, i}}{d x} u_{j}+\sum_{j=0}^{k-1} b_{j, i} \sum_{l=0}^{k-1} a_{l, j} u_{l}=\frac{n-k}{k} \sum_{j=0}^{k-1} b_{j, i} u_{j}+\sum_{j=0}^{k-1} a_{j, i} \sum_{l=0}^{k-1} b_{l, j} u_{l} .
$$

Therefore,

$$
\left(x \frac{d}{d x}-\frac{n-k}{k}\right) B=[B, A] .
$$

Assume the additional hypothesis $A$ is constant. We can assume that $A$ is the direct sum of $l$ Jordan blocks $A_{r}$ of size $m_{r}$ and eigenvalue $\lambda_{r}, 0 \leq$ $r \leq l-1$, where $1 \leq l \leq k$. Consider the block decomposition $B=\left(B_{r, s}\right)$, $0 \leq r, s \leq l-1, B_{r, s} \in \mathrm{M}_{m_{r} \times m_{s}}(\mathbb{C}\{x\})$. Let $A_{r}=\lambda_{r} I_{m_{r}}+N_{m_{r}}$ be the decomposition of the Jordan block $A_{r}$ into semisimple and nilpotent parts. 
We get a block decomposition $[B, A]=\left(B_{r, s} A_{s}-A_{r} B_{r, s}\right), B_{r, s} A_{s}-A_{r} B_{r, s}=$ $\left(\lambda_{s}-\lambda_{r}\right) B_{r, s}+B_{r, s} N_{m_{s}}-N_{m_{r}} B_{r, s}$. Hence

$$
\left(x \frac{d}{d x}+\lambda_{r}-\lambda_{s}-\frac{n-k}{k}\right) B_{r, s}=B_{r, s} N_{m_{s}}-N_{m_{r}} B_{r, s} .
$$

By Lemma 4.1 there are matrices $C_{r, s} \in \mathrm{M}_{m_{r} \times m_{s}}(\mathbb{C}), 0 \leq r, s \leq l-1$, such that

$$
B_{r, s}=C_{r, s} x^{\lambda_{s}-\lambda_{r}+\frac{n-k}{k}} .
$$

Hence $B_{r, s}=0$ or $\lambda_{s}-\lambda_{r}+\frac{n-k}{k} \in \mathbb{Z}$. Since we have assumed $2 \leq k \leq n-1$ and $(n, k)=1$,

$$
B_{r, r}=0 \quad \text { and } \quad B_{r_{0}, r_{1}}, B_{r_{1}, r_{2}}, \ldots, B_{r_{t-1}, r_{t}} \neq 0 \Rightarrow B_{r_{0}, r_{t}}=0,
$$

for $t=2, \ldots, l-1$. In particular $l \geq 2$. Since $B^{k}=(n / k)^{k} x^{n-k} I_{k}$, there are integers $i_{0}, \ldots, i_{k-1}$, s.t. $0 \leq i_{j} \leq l-1$ and $B_{i_{0}, i_{1}} B_{i_{1}, i_{2}} \ldots B_{i_{k-1}, i_{0}} \neq 0$. If $0 \leq$ $r<s \leq k-1, i_{r} \neq i_{s}$. Otherwise there would exist a constant matrix $C \neq 0$ s.t. $B_{i_{r}, i_{r+1}} \ldots B_{i_{s-1}, i_{s}}=x^{(s-r)(n-k) / k} C$ and $(s-r)(n-k) / k \in \mathbb{Z}$. Hence $l=k$ and the map $j \mapsto i_{j}$ defines a circular permutation of $\{0, \ldots, k-1\}$. We can assume $B_{j, i} \neq 0$ if $j \equiv i+1(\bmod k), i=0, \ldots, k-1$. By $(23)$ $B_{j, i}=0$ if $j \not \equiv i+1(\bmod k), i=0, \ldots, k-1$. Therefore $A$ is a diagonal matrix with eigenvalues $\lambda_{i}, 0 \leq i \leq k-1$. Moreover, $p u_{i}=C_{i+1, i} x^{\alpha_{i}} u_{i+1}$, where

$$
\alpha_{i}=\lambda_{i}-\lambda_{i+1}+\frac{n-k}{k}, i \in \mathbb{Z},
$$

and $\lambda_{j}=\lambda_{i}$ if $j \equiv i(\bmod ) k$. By Lemma 4.1, $\alpha_{i} \in \mathbb{Z}$. Since $\left(u_{0}, \ldots, u_{k-1}\right)$ is a basis of the $\mathbb{C}\{x\}$-module $L, \alpha_{i} \geq 0$. Up to a $\mathbb{C}$-linear change of basis, $C_{i+1, i}=n / k$ for $0 \leq i \leq k-1$.

Let us prove the proposition without the additional hypothesis $A$ is constant. If the eigenvalues of $A(0)$ do not differ by a nonzero integer we can assume $A=A(0)$ (see [24]) and the proposition is proved. In order to finish the proof it is enough to show that two eigenvalues of $A(0)$ cannot differ by a nonzero integer. Assume otherwise. Let $S$ be an invertible $\mathbb{C}\{x\}\left[x^{-1}\right]$-linear transformation of $\mathbb{C}\{x\}\left[x^{-1}\right] \otimes_{\mathbb{C}\{x\}} L$. We say that $S$ is a shearing transformation if there is a $\mathbb{C}\{x\}\left[x^{-1}\right]$-linear decomposition $\mathbb{C}\{x\}\left[x^{-1}\right] \otimes_{\mathbb{C}\{x\}} L=\oplus_{i \in I} L_{i}$ and a family of integers $m_{i}, i \in I$, such that $\left.S\right|_{L_{i}}=x^{m_{i}}$. Up to a shearing transformation of $\mathbb{C}\{x\}\left[x^{-1}\right] \otimes_{\mathbb{C}\{x\}} L$, we can assume that one of the eigenvalues of $A(0)$ has multiplicity bigger than one and that two eigenvalues of $A(0)$ do not differ by a nonzero integer. Hence we can assume $A=A(0)$. Therefore there are $i, j \in \mathbb{Z}, 1 \leq j \leq k-1$, such that $\lambda_{i}=\lambda_{i+j}$. By (24),

$$
j \frac{n-k}{k}=\alpha_{i}-\alpha_{i+j} \in \mathbb{Z} \text {. }
$$


Let $R$ denote the $\mathbb{C}$-algebra

$$
\mathbb{C}\left\{x, p, t_{1}, \ldots, t_{m-2}\right\} /\left(p^{k}-\left(\frac{n}{k}\right)^{k} x^{n-k}\right) .
$$

The derivation $x \partial_{x}+((n-k) / k) p \partial_{p}$ of $\mathbb{C}\left\{x, p, t_{1}, \ldots, t_{m-2}\right\}$ leaves invariant the ideal $\left(p^{k}-(n / k)^{k} x^{n-k}\right)$, inducing a derivation $\Delta$ of $R$.

Theorem 4.3. Let $L$ be a finitely generated torsion-free $R$-module of rank one. Let $\nabla$ be a $\mathbb{C}$-linear endomorphism of $L$ such that

$$
\nabla(f u)=\Delta(f) u+f \nabla u, f \in R, u \in L .
$$

If $\partial_{t_{j}}, 1 \leq j \leq m-2$, act on $L$ as $\mathbb{C}$-linear endomorphisms and

$$
\partial_{t_{j}}(f v)=\frac{\partial f}{\partial t_{j}} v+f \partial_{t_{j}} v, \quad 1 \leq j \leq m-2,
$$

for $f \in R$ and $v \in L, L$ is a free $\mathbb{C}\left\{x, t_{1}, \ldots, t_{m-2}\right\}$-module of rank $k$. Moreover, there is a system of generators of $L, v_{i}, i \in \mathbb{Z}$, and complex numbers $\lambda_{i}$, $i \in \mathbb{Z}$, such that $\left(v_{0}, \ldots, v_{k-1}\right)$ is a basis of $L$ as a $\mathbb{C}\left\{x, t_{1}, \ldots, t_{m-2}\right\}$-module, relation (1) holds and

$$
v_{i+k}=v_{i}, \quad \nabla v_{i}=\lambda_{i} v_{i}, \quad p v_{i}=\frac{n}{k} x^{\alpha_{i}} v_{i+1}, \quad \partial_{t_{j}} v_{i}=0, \quad 1 \leq j \leq m-2 .
$$

Proof. Assume in the first place that $m=2$. Since $L$ is a finitely generated $R$-module and $R$ is a finitely generated $\mathbb{C}\{x\}$-module, $L$ is a finitely generated $\mathbb{C}\{x\}$-module. Since $\mathbb{C}\{x\}$ is a principal ideal domain and $L$ is a torsion-free $\mathbb{C}\{x\}$-module, $L$ is a finitely free $\mathbb{C}\{x\}$-module. Let $l$ be the dimension of the $\mathbb{C}\{x\}$-module $L$.

Let $K$ be the quotient field of $R$. Since the rings $\mathbb{C}\{x\}\left[x^{-1}\right] \otimes_{\mathbb{C}\{x\}} R$ and $\mathbb{C}\{x\}\left[x^{-1}\right][p] /\left(p^{k}-(n / k)^{k} x^{n-k}\right)$ are isomorphic and $p^{k}-(n / k)^{k} x^{n-k}$ is an irreducible polynomial over the field $\mathbb{C}\{x\}\left[x^{-1}\right], \mathbb{C}\{x\}\left[x^{-1}\right] \otimes_{\mathbb{C}\{x\}} R$ is a field. Hence

$$
\mathbb{C}\{x\}\left[x^{-1}\right] \otimes_{\mathbb{C}\{x\}} R \stackrel{\rightrightarrows}{\rightarrow} K .
$$

Since $L$ has rank one, $K$ is a $\mathbb{C}\{x\}\left[x^{-1}\right]$-vector space of dimension $k$. By (29), $\mathbb{C}\{x\}\left[x^{-1}\right] \otimes_{\mathbb{C}\{x\}} L$ is a $\mathbb{C}\{x\}\left[x^{-1}\right]$-vector space of dimension $k$. Therefore $l=k$.

The action of $p$ on $L$ induces a $\mathbb{C}\{x\}$-linear endomorphism of $L$ such that $p^{k}=(n / k)^{k} x^{n-k}$. Since $[\nabla, p]=((n-k) / k) p$, the proof in the case $m=2$ follows from Theorem 4.2. Let us prove the theorem when $m \geq 3$.

Let $(t)$ denote the ideal of $R$ generated by $t_{1}, \ldots, t_{m-2}$. Set $\widetilde{L}=L /(t) L$. The $\mathbb{C}\{x, p\}$-module $\widetilde{L}$ verifies the assumptions of the theorem with $m=2$. Let $v_{i}, i \in \mathbb{Z}$, be a system of generators of $\mathbb{C}\{x\}$-module $\widetilde{L}$ in the conditions of Theorem 4.3. Take $w_{i} \in L$ such that $w_{i+k}=w_{i}$ and $v_{i}=w_{i}+(t) L$ for all $i$. Let $M$ be the $\mathbb{C}\left\{x, t_{1}, \ldots, t_{m-2}\right\}$-module generated by the $w_{i}$ 's. Since 
$L=M+(t)^{l} L$ for all $l, M=\cap_{l}\left(M+(t)^{l} L\right)=L$ (see [20], Proposition II.1.1.3).

We will denote (25) by $R_{m-2}$. We will show by induction that $w_{0}, \ldots, w_{k-1}$ are linearly independent over $R_{q}, q=0, \ldots, m-2$. The statement is true for $q=0$. Assume that it holds for a certain $q, 2 \leq q \leq m-3$. Assume that there are $a_{i} \in R_{q+1}$ such that $\sum_{i=0}^{k-1} a_{i} w_{i}=0$ and some of the $a_{i}$ 's do not vanish. There are a nonnegative integer $l$ and $i_{0} \in\{0, \ldots, k-1\}$ such that $a_{i} \in\left(t_{q+1}\right)^{l}, 0 \leq i \leq k-1$, and $a_{i_{0}} \notin\left(t_{q+1}\right)^{l+1}$. Since $L$ is torsion free,

$$
\sum_{i=0}^{k-1}\left(a_{i} / t_{q+1}^{l}\right) w_{i}=0 .
$$

Hence $\sum_{i=0}^{k-1}\left(\left(a_{i} / t_{q+1}^{l}\right)+\left(t_{q+1}\right)\right)\left(w_{i}+\left(t_{q+1}\right) L\right)=0$, contradicting the induction hypothesis. Hence $L$ is a free $\mathbb{C}\left\{x, t_{1}, \ldots, t_{m-2}\right\}$-module of rank $k$.

Let $c_{l, j, \nu} \in \mathbb{C}\left\{x, t_{1}, \ldots, t_{m-2}\right\}$ such that $\partial_{t_{j}} w_{l}=\sum_{\nu=0}^{k-1} c_{l, j, \nu} w_{l}$. Given $v=\sum_{l=0}^{k-1} a_{l} w_{l}, \partial_{t_{1}}$ annihilates $v$ if and only if

$$
\frac{\partial a_{l}}{\partial t_{1}}+\sum_{\nu=0}^{k-1} a_{\nu} c_{\nu, 1, l}=0, \quad l=0, \ldots, k-1 .
$$

Let $a_{1,0, l}, \ldots, a_{1, k-1, l} \in \mathbb{C}\left\{x, t_{1}, \ldots, t_{m-2}\right\}$ be the solution of (30) with Cauchy data $a_{1, \nu, l}(0)=\delta_{\nu, l}$. Here $\delta_{\nu, l}$ denotes the Kronecker symbol. Replacing $w_{l}$ by $\sum_{\nu=0}^{k-1} a_{1, \nu, l} w_{\nu}, l=0, \ldots, k-1$, we can assume from the beginning that $\partial_{t_{1}} w_{l}=0$ for all $l$. Now assume that $\partial_{t_{j}} w_{l}=0$ for $l=0, \ldots, k-1$, $j=1, \ldots, q(q \leq m-3)$. Let $c_{l, q+1, \nu} \in \mathbb{C}\left\{x, t_{1}, \ldots, t_{m-2}\right\}$ such that $\partial_{t_{q+1}} w_{l}=\sum_{\nu=0}^{k-1} c_{l, q+1, \nu} w_{\nu}$. Since $\partial_{t_{j}} w_{l}$ vanishes for all $j=1, \ldots, q$,

$$
\frac{\partial c_{l, q+1, \nu}}{\partial t_{j}}=0, \quad l, \nu=0, \ldots, k-1, \quad j=1, \ldots, q .
$$

By the argument above we can replace, $w_{l}, l=0, \ldots, k-1$, by $\sum_{\nu=0}^{k-1} a_{q+1, \nu, l} w_{\nu}$ with $a_{q+1, \nu, l} \in \mathbb{C}\left\{x, t_{q+1}, \ldots, t_{m-2}\right\}, a_{q+1, \nu, l}(0)=\delta_{\nu, l}$. Hence we can assume that $\partial_{t_{j}} w_{l}=0$ for $l=0, \ldots, k-1, j=1, \ldots, m-2$.

There are $\varepsilon_{i}, \delta_{i} \in(t) L$ such that

$$
\nabla w_{i}=\lambda_{i} w_{i}+\varepsilon_{i}, \quad p w_{i}=\frac{n}{k} x^{\alpha_{i}} w_{i+1}+\delta_{i}, \quad 0 \leq i \leq k-1 .
$$

Since $\partial_{t_{j}} w_{i}$ vanishes,

$$
\partial_{t_{j}} \varepsilon_{i}=\partial_{t_{j}}\left(\lambda_{i} w_{i}+\varepsilon_{i}\right)=\partial_{t_{j}}\left(\nabla w_{i}\right)=\nabla \partial_{t_{j}} w_{i}=0
$$

for $i \in \mathbb{Z}, j=1, \ldots, m-2$. A similar argument shows that $\partial_{t_{j}} \delta_{i}=0, i \in \mathbb{Z}$, $j=1, \ldots, m-2$.

The map that takes $x$ into $t^{k}, p$ into $\frac{n}{k} t^{n-k}$ and $t_{j}$ into $t_{j}$ for all $j$, identifies the integral closure of $R$ with the power series ring $\mathbb{C}\left\{t, t_{1}, \ldots, t_{m-2}\right\}$. Set $\mathcal{K}=\mathbb{C}\left\{t, t_{1}, \ldots, t_{m-2}\right\}\left[t^{-1}\right]$. Let $v: \mathcal{K} \rightarrow \mathbb{Z} \cup\{+\infty\}$ denote the canonical 
valuation by the order of the zero in the variable $t$. The semigroup of $R$ is by definition the additive sub-semigroup $\Gamma=(v(R) \backslash\{+\infty\})$ of $\mathbb{Z}$. A subset $\Sigma$ of $\mathbb{Z}$ is called a $\Gamma$-module if $\Gamma+\Sigma=\Sigma$. Two subsets $\Sigma_{1}, \Sigma_{2}$ of $\mathbb{Z}$ such that $\Gamma+\Sigma_{i}=\Sigma_{i}$ are isomorphic as $\Gamma$-modules if and only if there is an integer $\sigma$ verifying $\Sigma_{2}=\sigma+\Sigma_{1}$.

Given a torsion free $R$-module $L$ of rank one let $\phi: \mathcal{K} \otimes_{R} L \rightarrow \mathcal{K}$ be an isomorphism of $\mathcal{K}$-modules. Let $\Sigma_{\phi}$ denote the $\Gamma$-module defined by the intersection of $\mathbb{Z}$ with the image of the map $L \stackrel{-\otimes 1}{\longrightarrow} \mathcal{K} \otimes_{R} L \stackrel{\phi}{\longrightarrow} \mathcal{K} \stackrel{v}{\longrightarrow}(\mathbb{Z} \cup\{+\infty\})$. The set $\Sigma_{\phi}$ depends on $\phi$ but its isomorphism class as a $\Gamma$-module does not. We can choose $\phi$ in a way such that the minimum of $\Sigma_{\phi}$ equals 0 . We will denote this set by $\Sigma(L)$.

Given a family of nonnegative integers $\left(\alpha_{i}\right), i \in \mathbb{Z}$, such that (3) holds, denote by $L_{\left(\alpha_{i}\right)}$ the $R$-module generated by $v_{i}, i \in \mathbb{Z}$, with relations $v_{i+k}=v_{i}$ and $p v_{i}=\frac{n}{k} x^{\alpha_{i}} v_{i+1}$.

Proposition 4.4. Two R-modules $L_{\left(\alpha_{i}\right)}$ and $L_{\left(\beta_{i}\right)}$ are isomorphic if and only if there is $\nu \in \mathbb{Z}, 0 \leq \nu \leq k-1$, such that $\alpha_{i+\nu}=\beta_{i}$ for all $i \in \mathbb{Z}$.

Proof. The if part is clear. Let us prove the only if part. We can compute $\Sigma\left(L_{\left(\alpha_{i}\right)}\right)$ in the following way. Set $\gamma_{i}=v\left(\phi\left(v_{i}\right)\right)$. Since $v_{i+k}=v_{i}$ and $\phi\left(v_{i+1}\right)=\phi\left(v_{i}\right) \phi(p) \phi\left(\frac{n}{k} x^{\alpha_{i}}\right)^{-1}$,

$$
\gamma_{i+k}=\gamma_{i}, \quad \gamma_{i+1}=\gamma_{i}+n-k-k \alpha_{i}, \quad i \in \mathbb{Z} .
$$

Hence $\gamma_{i} \equiv \gamma_{j}(\bmod k)$ if and only if $i \equiv j(\bmod k)$. After performing a translation of $\mathbb{Z}$ and the replacement of $\phi$ by another isomorphism from $\mathcal{K} \otimes_{R} L$ onto $\mathcal{K}$, we can assume that $\gamma_{0}=\min \left\{\gamma_{i}: i \in \mathbb{Z}\right\}=0$. Under this assumption

$$
\gamma_{i}=(n-k) i-k\left(\alpha_{0}+\cdots+\alpha_{i-1}\right), \quad i \in \mathbb{Z} .
$$

If $w \in L_{\left(\alpha_{i}\right)} \backslash\{0\}$ there are $m_{i} \in \mathbb{Z}, f_{i} \in \mathcal{K}, 0 \leq i \leq k-1$, such that $m_{i} \geq 0$ and $w=\sum_{i=0}^{k-1} x^{m_{i}} f_{i} v_{i}$. Since $v\left(x^{m_{i}} f_{i}\right)$ is a multiple of $k$ or $v\left(x^{m_{i}} f_{i}\right)=+\infty$, $v\left(\phi\left(x^{m_{i}} f_{i} v_{i}\right)\right) \neq v\left(\phi\left(x^{m_{j}} f_{j} v_{j}\right)\right)$ if $i \neq j$. Therefore

$$
\gamma_{l}=\min \left\{j \in \Sigma\left(L\left(\alpha_{i}\right)\right): j \equiv(n-k) l(\bmod k)\right\} .
$$

It follows from (32) and (33) that we can recover the family $\left(\alpha_{i}\right)$ from $\Sigma\left(L_{\left(\alpha_{i}\right)}\right)$.

Proposition 4.5. A torsion free $R$-module $L$ of rank 1 is free if and only if $\Sigma(L)=\Gamma$. In particular, $L_{\left(\alpha_{i}\right)}$ is free if and only if there is one and only one $i \in\{0, \ldots, k-1\}$ such that $\alpha_{i} \neq 0$.

Proof. If the $R$ module $L$ is generated by $u, \Sigma_{\phi}$ is generated by an integer $l$. Let $u$ be an element of $L$ with valuation $l$. Let us show that $L$ equals $R u$. We will identify $\mathcal{K}$ with $\mathbb{C}\left\{x, p, t_{1}, \ldots, t_{m-2}\right\}\left[x^{-1}\right]$. We will identify $L$ with 
its image on $\mathcal{K}$. If $w \in L$, there are $a_{i} \in \mathbb{C}\left\{x, t_{1}, \ldots, t_{m-2}\right\} \backslash(x)$ and $r_{i} \in \mathbb{Z}$, $0 \leq i \leq k-1$, such that $w=\sum_{i=0}^{k-1} a_{i} p^{i} x^{r_{i}} u$. Hence

$$
v(w)=v(u)+\inf \left\{(n-k) i+k r_{i}: a_{i} \neq 0\right\} .
$$

Since $v(w) \in \Sigma_{\phi}, r_{i} \geq 0$. Hence $w \in L$.

Theorem 4.6. Let $X$ be a complex manifold of dimension $m$. Let $\Lambda$ be the germ at $q \in T^{*} X$ of an irreducible conic Lagrangian variety contained in an involutive submanifold of $T^{*} X \backslash X$ of codimension $m-1$. Given a system of microdifferential equations $\mathcal{M}$ of multiplicity one along $\Lambda$, there are complex numbers $\lambda_{i}, i \in \mathbb{Z}$ such that (1) holds and after a convenient quantized contact transformation the germ at $q$ of $\mathcal{M}$ is isomorphic to $\mathcal{M}_{\left(\lambda_{i}\right)}$. Two systems $\mathcal{M}_{\left(\lambda_{i}\right)}$ and $\mathcal{M}_{\left(\mu_{i}\right)}$ are isomorphic if and only if there is $\nu \in$ $\{0, \ldots, k-1\}$, such that

$$
\beta_{i}=\alpha_{i+\nu}, \quad i \in \mathbb{Z}, \quad \text { and } \quad \mu_{0} \equiv \lambda_{\nu}\left(\bmod \frac{n}{k}\right),
$$

where $\beta_{i}=\mu_{i}-\mu_{i+1}+(n-k) / k$.

Proof. Let $X$ be a copy of $\mathbb{C}^{m}$ with coordinates $\left(x, y, t_{1}, \ldots, t_{m-2}\right)$. On a neighborhood of $(0, d y)$ the canonical 1-form $\theta$ of $T^{*} X$ equals $\xi d x+\eta d y+$ $\sum_{i=1}^{m-2} \tau_{i} d t_{i}=\eta\left(d y-\left(p d x+q_{1} d t_{1}+\cdots+q_{m-2} d t_{m-2}\right)\right)$. The conormal of the hypersurface of $\mathbb{C}^{m}$ with equation $y^{k}-x^{n}=0$ equals the Lagrangian variety defined by the equations

$$
y-\frac{k}{n} x p=p^{k}-\left(\frac{n}{k}\right)^{k} x^{n-k}=q_{1}=\cdots=q_{m-2}=0 .
$$

Following [18], Theorem 8.3, we can assume that the support of $\mathcal{M}$ equals (35). Let $\mathcal{N}$ be the canonical lattice of $\mathcal{M}$. The fiber at $(0, d y)$ of $\mathcal{O}_{\Lambda}(0)$ equals $R$. Let $M, N$ and $L$ denote, respectively, the fibers at $(0, d y)$ of the sheaves $\mathcal{M}, \mathcal{N}$ and $\mathcal{N} / \mathcal{N}(-1)$. By Proposition $2.7 L$ is a finitely generated torsion free $R$-module of rank one.

Since $\left[\vartheta, \mathcal{E}_{X}(0)\right] \subset \mathcal{E}_{X}(0),\left[\vartheta, \mathcal{I}_{\Lambda}(-1)\right] \subset \mathcal{I}_{\Lambda}(-1)$ and $(6)$ holds, the operator $\vartheta$ acts on $R$ as a derivation by

$$
\vartheta(f)=\sigma_{0}([\vartheta, P])=\left\{\sigma(\vartheta), \sigma_{0}(P)\right\}=H_{\sigma(\vartheta)}(f),
$$

where $P \in \mathcal{E}_{X}(0)$ such that $\sigma_{0}(P)=f$. Here $\{\cdot, \cdot\}$ denotes the Poisson brackets. Since

$$
H_{\sigma(\vartheta)}=x \partial_{x}+\frac{n}{k} y \partial_{y}+\frac{n-k}{k} p \partial_{p}-\eta \partial_{\eta},
$$

$H_{\sigma(\vartheta)}$ acts on $\mathbb{C}\left\{x, p, t_{1}, \ldots, t_{m-2}\right\}$ as the $x \partial_{x}+\frac{n-k}{k} p \partial_{p}$-derivation. Hence $\vartheta$ acts on $R$ as the derivation $\Delta$. Moreover,

$$
[\vartheta, p]=H_{\sigma(\vartheta)}(p)=\frac{n-k}{k} p .
$$


By the regularity conditions $\vartheta \mathcal{N}(k) \subset \mathcal{N}(k)$ and $\partial_{t_{j}} \mathcal{N}(k) \subset \mathcal{N}(k)$ for $k \in \mathbb{Z}$ and $1 \leq j \leq m-2$. If $u \in \mathcal{N}, v=u+\mathcal{N}(-1), P \in \mathcal{E}_{X}(0)$ and $f=\sigma_{0}(P)$, $\vartheta P u=[\vartheta, P] u+P \vartheta u$. Setting $\nabla=\vartheta$ we deduce that (26) holds. A similar argument shows that (27) holds.

We have shown that $L$ verifies the hypothesis of Theorem 4.3. Let $v_{i}$, $i \in \mathbb{Z}$, be a system of generators of $L$ verifying (28). Choose $\widetilde{v}_{i} \in N, i \in \mathbb{Z}$, such that $\widetilde{v}_{i+k}=\widetilde{v}_{i}$ and $v_{i}=\widetilde{v}_{i}+N(-1)$. The $\widetilde{v}_{i}$ 's generate the $\mathcal{E}_{X,(0, d y)}(0)-$ module $N$. For $i \in \mathbb{Z}$ and $l=1, \ldots, m-2$, set

$$
\omega_{i, l}=\partial_{t_{l}} \widetilde{v}_{i}, \quad \omega_{i}=\left(\partial_{x} \partial_{y}^{-1}\right) \widetilde{v}_{i}-\left(-\frac{n}{k}\right) x^{\alpha_{i}} \widetilde{v}_{i+1} .
$$

By the microdifferential Cauchy Theorem I. 6.1.1 of [20] we can assume that $\omega_{i, l}=0$ for $i \in \mathbb{Z}, l=1, \ldots, m-2 . l \geq 0 N(-l)=\mathcal{E}_{X,(0, d y)}(-l) N$. We will show that $\omega_{i} \in N_{(-l)}$ for all $l \geq 1, i \in \mathbb{Z}$. We know that $\omega_{i} \in N_{(-1)}$ for all $i$. Assume that $\omega_{i} \in N(-l)$. Since $L$ is a free $\mathbb{C}\left\{x, t_{1}, \ldots, t_{m-2}\right\}$-module of rank $k$, there are $a_{i, j} \in \mathbb{C}\left\{x, t_{1}, \ldots, t_{m-2}\right\}, \varpi_{i} \in N(-l-1)$ such that

$$
\omega_{i}=\sum_{j=0}^{k-1} a_{i, j} \partial_{y}^{-l} \widetilde{v}_{j}+\varpi_{i} .
$$

Since $\partial_{t_{s}} \omega_{i}=\sum_{j=0}^{k-1}\left(\partial a_{i, j} / \partial t_{s}\right) \partial_{y}^{-l} \widetilde{v}_{j}+\partial_{t_{s}} \varpi_{i}$ vanishes for all $s, a_{i, j}$ lies in $\mathbb{C}\{x\}$ for all $i, j$. In one hand there is $\varpi_{i}^{\prime} \in N(-l-1)$ such that

$$
\begin{aligned}
\vartheta \omega_{i} & =\left(\partial_{x} \partial_{y}^{-1}\right)\left(\lambda_{i}+\frac{n-k}{k}\right) \widetilde{v}_{i}-\left(-\frac{n}{k}\right) x^{\alpha_{i}}\left(\lambda_{i+1}+\alpha_{i}\right) \widetilde{v}_{i+1} \\
& =\left(\lambda_{i}+\frac{n-k}{k}\right)\left(\left(\partial_{x} \partial_{y}^{-1}\right) \widetilde{v}_{i}-\left(-\frac{n}{k}\right) x^{\alpha_{i}} \widetilde{v}_{i+1}\right) \\
& =\left(\lambda_{i}+\frac{n-k}{k}\right)\left(\sum_{j=0}^{k-1} a_{i, j} \partial_{y}^{-l} \widetilde{v}_{j}\right)+\varpi_{i}^{\prime} .
\end{aligned}
$$

On the other hand there is $\varpi_{i}^{\prime \prime} \in N(-l-1)$ such that

$$
\vartheta \omega_{i}=\sum_{j=0}^{k-1}\left(x \frac{\partial}{\partial x}+\frac{n}{k} l+\lambda_{i}\right) a_{i, j} \partial_{y}^{-l} \widetilde{v}_{j}+\varpi_{i}^{\prime \prime} .
$$

It follows from (36) and (37) that $a_{i, j} \in \mathbb{C}\{x\}$ is annihilated by the differential operator $x \frac{\partial}{\partial x}+1+\frac{n(l-1)}{k}$. Hence $a_{i, j}$ vanishes for all $i, j$. This implies that $\omega_{i} \in N(-l-1)$.

We can assume that $\alpha_{-1} \neq 0$. Let $\iota: \mathbb{Z} \rightarrow\left\{i: \alpha_{i-1} \neq 0\right\}$ be the unique increasing bijection verifying $\iota(0)=0$. For $s \in \mathbb{Z}$ set $m_{s}=\iota(s+1)-\iota(s)$, $\beta_{s}=\alpha_{\iota(s+1)-1}$ and $\lambda_{s}^{\prime}=(k / n) \lambda_{\iota(s)}$. Set $d=\#\left\{i+k \mathbb{Z}: \alpha_{i-1} \neq 0\right\}$. Then $m_{s+d}=m_{s}, \beta_{s+d}=\beta_{s}, \sum_{s=0}^{d-1} m_{s}=k, \sum_{s=0}^{d-1} \beta_{s}=n-k$ and $\lambda_{s+1}^{\prime}-\lambda_{s}^{\prime}=$ 
$(1 / n)\left((n-k) m_{s}-k \beta_{s}\right)$. Since $\omega_{i}$ vanish for all $i$, the $\mathcal{E}_{X,(0, d y)}(0)$-module $N$ is generated by $\widetilde{v}_{\iota(s)}, s \in \mathbb{Z}$. Moreover, for $s \in \mathbb{Z}, l=1, \ldots, m-2$,

$$
\left(\partial_{x} \partial_{y}^{-1}\right)^{m_{s}} \widetilde{v}_{\iota(s)}=\left(-\frac{n}{k}\right)^{m_{s}} x^{\beta_{s}} \widetilde{v}_{\iota(s+1)}, \quad \partial_{t_{l}} \widetilde{v}_{\iota(s)}=0 .
$$

Let us prove that $\vartheta$ is given by a diagonal matrix with respect to a convenient system of generators of the $\mathcal{E}_{X,(0, d y)}(0)$-module $N$. Set $\vartheta^{\prime}=(k / n) \vartheta$. Then $\left(\vartheta^{\prime}-\lambda_{s}^{\prime}\right) \widetilde{v}_{\iota(s)} \in N(-1)$ for all $s$. Let $A_{0}$ be the matrix $\operatorname{diag}\left(\lambda_{0}^{\prime}, \ldots, \lambda_{d-1}^{\prime}\right)$. There is a matrix $\mathcal{A}_{-1} \in \mathrm{M}_{d}\left(\mathcal{E}_{X(-1)}\right)$ such that $\left(\vartheta^{\prime}-A_{0}-\mathcal{A}_{-1}\right) \widetilde{v}_{\iota(s)}=0$. If $d=1$ we can assume, by Lemma 8.8 of [18] (or Theorem 3.1 of [17]), that $\mathcal{A}_{-1}$ vanishes. Assume that $d>1$. By construction $1 \leq m_{s} \leq k-1$, $1 \leq \beta_{s} \leq n-k-1, \sum_{s=0}^{d-1} m_{s}=k, \sum_{s=0}^{d-1} \beta_{s}=n-k$. Since

$$
\lambda_{s+l}^{\prime}-\lambda_{s}^{\prime}=\frac{1}{n}\left((n-k) \sum_{j=s}^{s+l-1} m_{j}-k \sum_{j=s}^{s+l-1} \beta_{j}\right), \quad 0 \leq l \leq d-1,
$$

$\left|\lambda_{s+l}^{\prime}-\lambda_{s}^{\prime}\right| \in(1 / n)(k \mathbb{N}+(n-k) \mathbb{N})$ if and only if $l=0$ (see [3], Lemma 10). By Theorem 3.5 we can assume that $\mathcal{A}_{-1}$ vanishes. For $i \in \mathbb{Z}, \iota(s) \leq i<\iota(s+1)$, set $u_{i}=\left(-\partial_{x} \partial_{y}^{-1}\right)^{i-\iota(s)} \widetilde{v}_{\iota(s)}$. The $\mathcal{E}_{X,(0, d y)}$-module $M$ is generated by $u_{i}$, $i \in \mathbb{Z}$, and verifies the relations (1) and (2).

Let us prove the second statement of the theorem.

By Theorem 2.5 the restriction of $\mathcal{M}_{\left(\lambda_{i}\right)}$ to the regular locus of $\Lambda$ has simple characteristics. We are going to compute the degree of homogeneity of the principal symbol of this restriction (see $\S 3$ of [18] for details).

We will identify the regular locus of $\Lambda$ with $\mathbb{C}^{*} \times \mathbb{C}^{*} \times \mathbb{C}^{m-2}$ by the parametrization $\gamma: \mathbb{C}^{*} \times \mathbb{C}^{*} \times \mathbb{C}^{m-2} \rightarrow \Lambda$ given by

$$
\gamma\left(t, \tau, t_{1}, \ldots, t_{m-2}\right)=\left(t^{k}, t^{n}, t_{1}, \ldots, t_{m-2} ;-(n / k) t^{n-k} \tau, \tau, 0, \ldots, 0\right) .
$$

We have

$$
\begin{aligned}
t \partial_{t} & =k x \partial_{x}+n y \partial_{y}+(n-k) \xi \partial_{\xi} \\
\tau \partial_{\tau} & =\xi \partial_{\xi}+\eta \partial_{\eta} .
\end{aligned}
$$

Set $u=u_{0}, \lambda=\lambda_{0}$ and set, for $x \neq 0$,

$$
\begin{gathered}
P=k x \partial_{x}+n y \partial_{y}-k \lambda \\
Q=x^{n-k}\left(\prod_{i=1}^{k}\left(x^{-\alpha_{k-i}} \partial_{x}\right)-\left(-\frac{n}{k}\right)^{k} \partial_{y}^{k}\right) .
\end{gathered}
$$

It follows from (2) that, outside of $\{x=0\}$, the differential operators $P, Q$ and $\partial_{t_{j}}, j=1, \ldots, m-2$, annihilate $u$. Set $\alpha=\sum_{j=0}^{k-2} \sum_{i=0}^{j} \alpha_{i}$. Then

$$
\sigma_{k}(Q)=\xi^{k}-\left(-\frac{n}{k}\right)^{k} x^{n-k} \eta^{k}, \quad \sigma_{k-1}(Q)=-\frac{\alpha}{x} \xi^{k-1} .
$$


The principal symbol of $u$ is a solution of the homogeneous system defined by the operators

$$
\begin{aligned}
\widetilde{L}_{P} & =t \partial_{t}-n \tau \partial_{\tau}-k \lambda+\frac{1}{2}(1-k)-n \\
\widetilde{L}_{Q} & =\frac{\xi^{k-1}}{x}\left(t \partial_{t}-\alpha+\frac{1}{2}(n-k-1)(k-1)\right) .
\end{aligned}
$$

Therefore the degree of homogeneity of the principal symbol of $u$ equals

$$
\frac{1}{n}\left(\alpha-\lambda k-\frac{1}{2}(n-k)(k-1)\right) \quad(\bmod \mathbb{Z}),
$$

that is, equals,

$$
-\frac{1}{n} \sum_{i=0}^{k-1} \lambda_{i} \quad(\bmod \mathbb{Z})
$$

Actually,

$$
\sum_{i=0}^{k-1} \lambda_{i}=k \lambda_{0}-\alpha+\frac{n-k}{k} \sum_{i=1}^{k-1} i=k \lambda_{0}-\alpha+\frac{(n-k)(k-1)}{2} .
$$

By Theorem 4.1 of $[8]$ the congruence class (modulo $\mathbb{Z}$ ) of the degree of homogeneity of the section $u \neq 0$ determines the structure of the restriction of $\mathcal{M}_{\left(\lambda_{i}\right)}$ to the regular locus of $\Lambda$. Hence this congruence class does not depend on the choice of the generator $u$. Therefore, if $\mathcal{M}_{\left(\lambda_{i}\right)} \simeq \mathcal{M}_{\left(\mu_{i}\right)}$,

$$
\alpha-k \lambda_{0} \equiv \beta-k \mu_{0} \quad(\bmod n \mathbb{Z}),
$$

where $\beta=\sum_{j=0}^{k-2} \sum_{i=0}^{j} \beta_{i}$. The module $\mathcal{N}=\sum_{i} \mathcal{E}_{X}(0) u_{i}$ satisfies the conditions of Theorem 2.3. Therefore the $R$-module canonically associated by Theorem 2.3 to the system $\mathcal{M}_{\left(\lambda_{i}\right)}$ equals the $R$-module $L_{\left(\alpha_{i}\right)}$ introduced in Proposition 4.4. By Theorem 2.3 the $R$-modules $L_{\left(\alpha_{i}\right)}$ and $L_{\left(\beta_{i}\right)}$ are isomorphic. By Proposition 4.4 we can assume that there is a $\nu \in \mathbb{Z}, 0 \leq \nu \leq k-1$, such that $\beta_{i}=\alpha_{i+\nu}$ for all $i \in \mathbb{Z}$. Notice that

$$
\sum_{j=0}^{k-2} \sum_{i=0}^{j}\left(\alpha_{i+\nu}-\alpha_{i}\right)=\nu(n-k)-k \sum_{i=0}^{\nu-1} \alpha_{i}=k\left(\lambda_{\nu}-\lambda_{0}\right) .
$$

By the previous relation along with $(40), \mu_{0}-\lambda_{\nu} \equiv 0\left(\bmod \frac{n}{k}\right)$.

Conversely, assume that $\beta_{i}=\alpha_{i+\nu}$ and $\mu_{0}=\lambda_{\nu}+l \frac{n}{k}$. The inner automorphism of $\mathcal{E}_{X}, R \mapsto \partial_{y}^{-l} R \partial_{y}^{l}$, changes $\vartheta-\mu_{i}$ into $\vartheta-\lambda_{i}$ for all $i$. Hence $\mathcal{M}_{\left(\lambda_{i}\right)} \simeq \mathcal{M}_{\left(\mu_{i}\right)}$. 


\section{D-modules.}

Let $\Lambda$ be a germ at a point $q \in T^{*} X \backslash T_{X}^{*} X$ of a conic Lagrangian variety in generic position. Let $\mathcal{L}$ be a germ at $\pi(q)$ of a coherent $\mathcal{D}_{X}$-module with characteristic variety contained in the union of $\Lambda$ with the zero section. We say that $\mathcal{L}$ belongs to the category $\mathcal{H} o l(\Lambda, \mathcal{D})$ if each germ at $\pi(q)$ of a vector field $\omega$ such that $\sigma(\omega)(q) \neq 0$ induces an isomorphism of complex vector spaces $u: \mathcal{L}_{\pi(q)} \rightarrow \mathcal{L}_{\pi(q)}$. Here $\sigma(\omega)(q)$ denotes the principal symbol of the differential operator $\omega$. Let $\mathcal{H o l}(\Lambda, \mathcal{E})$ denote the category of germs at $q$ of coherent $\mathcal{E}_{X}$-modules with characteristic variety contained in $\Lambda$.

Theorem 5.1 (See [2], Theorem 8.6.19). The functor $\mu_{q}: \mathcal{H o l}(\Lambda, \mathcal{D}) \rightarrow$ $\mathcal{H o l}(\Lambda, \mathcal{E})$, defined by $\mu_{p}(\mathcal{L})=\mathcal{E}_{X, q} \otimes_{\mathcal{D}_{X, \pi(q)}} \mathcal{L}$, is an equivalence of categories. Its quasi-inverse is the base change functor associated to the inclusion morphism $\mathcal{D}_{X, \pi(q)} \hookrightarrow \mathcal{E}_{X, q}$.

Given a ring $R$ and $\varepsilon \in R$, we use Pochhammer's notation

$$
(\varepsilon)_{j}=\varepsilon(\varepsilon+1) \ldots(\varepsilon+j-1) .
$$

Theorem 5.2. Let $k, n$ be integers such that $2 \leq k \leq n-1$ and $(k, n)=1$. Let $\mathcal{L}$ be the germ at the origin of a coherent $\mathcal{D}_{\mathbb{C}^{m} \text {-module with characteristic }}$ variety equal to the union of the conormal of the hypersurface $y^{k}=x^{n}$ with the zero section. Then $\mathcal{L}$ has multiplicity one along the conormal of $y^{k}=x^{n}$ and

$$
\partial_{y}: \mathcal{L}_{0} \rightarrow \mathcal{L}_{0}
$$

is an isomorphism of complex vector spaces if and only if there are nonnegative integers $\alpha_{i}, i \in \mathbb{Z}$, and complex numbers $\lambda_{i}, i \in \mathbb{Z}$, such that $\mathcal{L}$ is isomorphic to $\mathcal{L}_{\left(\lambda_{i}\right)}$ and

$$
\lambda_{i} \notin \frac{n}{k}\{-1,-2, \ldots\}, \quad 0 \leq i \leq k-1 .
$$

Proof. Let us show that Condition (43) is necessary. By Theorem 8.6.19 of [2] and Theorem 4.6, $\mathcal{L}$ is isomorphic to some $\mathcal{D}$-module $\mathcal{L}_{\left(\lambda_{i}\right)}$. Set $q=$ $(0, d y) \in T^{*} \mathbb{C}^{m}$. Set $t=\left(t_{1}, \ldots, t_{m-2}\right)$. It follows from (2) that

$$
y \partial_{y} u_{l}=\frac{k}{n} \lambda_{l} u_{l}+x^{\alpha_{l}+1} \partial_{y} u_{l+1}, \quad \partial_{t_{j}} u_{l}=0,
$$

$l=0, \ldots, k-1, j=1, \ldots, m-2$. It follows from (44) and (2) that we have an isomorphism of complex vector spaces

$$
\left(\mathcal{L}_{\left(\lambda_{i}\right)}\right)_{0} \cong \bigoplus_{i=0}^{k-1}\left(\mathbb{C}\{x, t\}\left[\partial_{y}\right] \oplus y \mathbb{C}\{x, y, t\}\right) u_{i} .
$$

Set $V=\oplus_{l=0}^{k-1}\left(\mathbb{C}\{x, t\}\left[\partial_{y}\right] \oplus y \mathbb{C}\{x, t\}[y]\right) u_{l}$. Set $\delta_{l, i}=i+\alpha_{l}+\cdots+\alpha_{l+i-1}$, $0 \leq l \leq k-1, i \geq 0$. Assume that (42) is injective. We will show by 
induction in $r$ that $\lambda_{l} \notin(n / k)\{-1,-2, \ldots,-r\}$. Set

$$
\begin{gathered}
Q_{j, l}=x^{\delta_{l, j+1}} u_{l+j+1}+\sum_{i=1}^{j} k \frac{\lambda_{l+i}}{n} x^{\delta_{l, i}} R_{j-i, l+i}, \\
R_{j, l}=\left(k \frac{\lambda_{l}}{n}+j+1\right)^{-1}\left(y^{j+1} u_{l}-Q_{j, l}\right),
\end{gathered}
$$

for $0 \leq l \leq k-1,0 \leq j \leq r$. Since

$$
\partial_{y}\left(y^{r+1} u_{l}-Q_{r, l}\right)=\left(k \frac{\lambda_{l}}{n}+r+1\right) y^{r} u_{l}
$$

and $Q_{r, l}$ is a $\mathbb{C}\{x, t\}$-linear combination of $y^{j} u_{l}, 0 \leq j \leq r, 0 \leq l \leq k-1$,

$$
k \frac{\lambda_{l}}{n}+r+1 \neq 0
$$

Hence we can define $R_{r+1, l}$ and $Q_{r+1, l}$ and iterate the procedure.

Assume that (43) holds. We can show by induction in $s \in \mathbb{N}$ that there are complex numbers $b_{l, r, s}$ such that

$$
\left(\partial_{y} y\right)_{s} u_{l}=\left(k \frac{\lambda_{l}}{n}+1\right)_{s} u_{l}+\sum_{r=1}^{s} b_{l, r, s} x^{\delta_{l, r}} \partial_{y}^{r} u_{l+r} .
$$

Since $\left(\partial_{y} y\right)_{s}=\left(\partial_{y}\right)^{s} y^{s}$,

$$
\left(k \frac{\lambda_{l}}{n}+1\right)_{s} \partial_{y}^{-s} u_{l}=y^{s} u_{l}-\sum_{r=1}^{s} b_{l, r, s} x^{\delta_{l, r}} \partial_{y}^{r-s} u_{l+r} .
$$

By (43) there are complex numbers $a_{l, r, s}$ such that

$$
\partial_{y}^{-s} u_{l}=\sum_{r=0}^{s} a_{l, r, s} x^{\delta_{l, r}} y^{s-r} u_{l+r} .
$$

Let $W_{-s}, s \in \mathbb{N}$, be the $\mathbb{C}\{x, y, t\}$-submodule of $\oplus_{l=0}^{k-1} \mathbb{C}\{x, y, t\} u_{l}$ generated by $\partial_{y}^{-s} u_{l}, l=0, \ldots, k-1$. Let $V_{-s}, s \in \mathbb{N}$, be the $\mathbb{C}\{x, t\}[y]$-submodule of $V$ generated by $\partial_{y}^{-s} u_{l}, l=0, \ldots, k-1$. By $(51)$,

$$
\bigoplus_{l=0}^{k-1} \mathcal{E}_{\mathbb{C}^{m}, q}(-s) u_{l} \subset W_{-s}+\bigoplus_{l=0}^{k-1} \mathcal{E}_{\mathbb{C}^{m}, q}(-s-1) u_{l},
$$

for all $s \geq 0$. Hence

$$
\bigoplus_{l=0}^{k-1} \mathcal{E}_{\mathbb{C}^{m}, q}(-s) u_{l} \subset W_{0}+\bigoplus_{l=0}^{k-1} \mathcal{E}_{\mathbb{C}^{m}, q}(-s) u_{l},
$$


for all $s \geq 0$. By Proposition II.1.1.3 of [20],

$$
\bigoplus_{l=0}^{k-1} \mathbb{C}\{x, y, t\} u_{l}=\bigoplus_{l=0}^{k-1} \mathcal{E}_{\mathbb{C}^{m}, q}(0) u_{l}
$$

Hence the inclusion

$$
\left(\mathcal{L}_{\left(\lambda_{i}\right)}\right)_{0} \hookrightarrow\left(\mathcal{M}_{\left(\lambda_{i}\right)}\right)_{q}
$$

is surjective. Let $\Phi$ denote the $\mathbb{C}\{x, t\}$-linear endomorphism of $V$ defined by $\Phi\left(\partial_{y}^{j+1} u_{l}\right)=\partial_{y}^{j} u_{l}, \Phi\left(y^{j} u_{l}\right)=R_{j, l}, j \geq 0$. Notice that (42) induces a $\mathbb{C}\{x, t\}$-linear endomorphism of $V$. Moreover,

$$
\partial_{y} V_{-s} \subset V_{-s+1}, \partial_{y} W_{-s} \subset W_{-s+1} \text { and } \Phi\left(V_{-s}\right) \subset V_{-s-1} .
$$

By $(48), \Phi\left(\partial_{y} y^{j+1} u_{l}\right)=$

$$
\begin{aligned}
& =\Phi\left(\partial_{y} Q_{j, l}+\left(k \frac{\lambda_{l}}{n}+j+1\right) y^{j} u_{l}\right) \\
& \left.=\Phi\left(\partial_{y}\left(x^{\delta_{l, j+1}} u_{l+j+1}+\sum_{i=1}^{j} k \frac{\lambda_{l+i}}{n} x^{\delta_{l, i}} R_{j-i, l+i}\right)\right)+\left(k \frac{\lambda_{l}}{n}+j+1\right) y^{j} u_{l}\right) \\
& =x^{\delta_{l, j+1}} u_{l+j+1}+\sum_{i=1}^{j} k \frac{\lambda_{l+i}}{n} x^{\delta_{l, i}} R_{j-i, l+i}+y^{j+1} u_{l}-Q_{j, l} \\
& =y^{j+1} u_{l} .
\end{aligned}
$$

Therefore the kernel of (42) is contained in $W_{-s}$ for all $s$. Hence (42) is injective. By (53) and (47) $\partial_{y} \Phi\left(y^{j} u_{l}\right)=y^{j} u_{l}$ for $0 \leq l \leq k-1, j \geq 0$. Hence $\partial_{y} \mathcal{L}_{0}+W_{-s}=\mathcal{L}_{0}$ for all $s$. By Proposition II.1.1.3 of [20], (42) is surjective. The result follows from Theorem 5.1.

The higher hypergeometric series was introduced by Thomae (cf. [23]) as the series

$$
{ }_{k} F_{k-1}\left(\varepsilon_{0}, \ldots, \varepsilon_{k-1}, \theta_{0}, \ldots, \theta_{k-2} \mid z\right)=\sum_{j=0}^{\infty} \frac{\left(\varepsilon_{0}\right)_{j} \ldots\left(\varepsilon_{k-1}\right)_{j} z^{j}}{\left(\theta_{0}\right)_{j} \ldots\left(\theta_{k-2}\right)_{j} j !} .
$$

Set $\delta_{z}=z \frac{d}{d z}$. Given $\widetilde{\alpha}=\left(\widetilde{\alpha}_{i}\right), \widetilde{\beta}=\left(\widetilde{\beta}_{i}\right), i=0, \ldots, k-1$, set

$$
D(\widetilde{\alpha}, \widetilde{\beta})=\left(\delta_{z}+\widetilde{\beta}_{0}-1\right) \ldots\left(\delta_{z}+\widetilde{\beta}_{k-1}-1\right)-z\left(\delta_{z}+\widetilde{\alpha}_{0}\right) \ldots\left(\delta_{z}+\widetilde{\alpha}_{k-1}\right) .
$$

If the $\widetilde{\beta}_{i}$ 's are distinct modulo $\mathbb{Z}$ then $k$ independent solutions of $D(\widetilde{\alpha}, \widetilde{\beta}) \varphi=$ 0 are given by

$$
z^{1-\widetilde{\beta}_{i}} F_{k-1}\left(1+\widetilde{\alpha}_{0}-\widetilde{\beta}_{i}, \ldots, 1+\widetilde{\alpha}_{k-1}-\widetilde{\beta}_{i}, 1+\widetilde{\beta}_{0}-\widetilde{\beta}_{i}, \ldots, 1+\widetilde{\beta}_{k-1}-\widetilde{\beta}_{i} \mid z\right)
$$

for $i=0, \ldots, k-1$. Here $\ldots$ denotes the omission of $1+\widetilde{\beta}_{i}-\widetilde{\beta}_{i}$. Levelt computed the monodromy of the equations above (see $[\mathbf{1 1}],[\mathbf{1}]$ ). 
For $i, j=0, \ldots, k-1$, set $\widetilde{\alpha}_{j}=\left(\sum_{0 \leq l \leq j-1} \alpha_{l}+j\right) / n, \widetilde{\beta}_{j}-1=\lambda / n-j / k$, $\varepsilon_{i, j}=1+\widetilde{\alpha}_{j}-\widetilde{\beta}_{i}$ and $\theta_{i, j}=1+\widetilde{\beta}_{j}-\widetilde{\beta}_{i}$.

Theorem 5.3. We have $k$ independent solutions of $\mathcal{L}_{\left(\lambda_{i}\right)}$ given by the analytic continuations of

$$
y^{\lambda_{0} \frac{k}{n}}\left(\frac{y^{k}}{x^{n}}\right)^{1-\widetilde{\beta}_{i}}{ }_{k} F_{k-1}\left(\varepsilon_{i, 0}, \ldots, \varepsilon_{i, k-1}, \theta_{i, 0}, \ldots, \check{\theta}_{i, i}, \ldots, \theta_{i, k-1} \mid \frac{y^{k}}{x^{n}}\right),
$$

for $i=0, \ldots, k-1$.

Proof. Set $\lambda=\lambda_{0}, u=u_{0}$ and $v(x, y)=y^{-\lambda \frac{k}{n}} u(x, y)$. Since $\vartheta v=0, v$ is constant along the integral curves of $\vartheta$, that is, along the fibers of the map $\Phi: \mathbb{C}^{2} \backslash\{(0,0)\} \rightarrow \mathbb{P}^{1}$ defined by $\gamma(x, y)=\left(x^{n}: y^{k}\right)$. Since $v$ is a multivalued holomorphic function ramified along $x y\left(y^{k}-x^{n}\right)=0$, there is a multivalued holomorphic function $\varphi$ on $\mathbb{P}^{1}$, ramified along $0,1, \infty$, such that $v=\varphi \circ \Phi$. Hence,

$$
u(x, y)=y^{\lambda \frac{k}{n}} \varphi\left(\frac{y^{k}}{x^{n}}\right) .
$$

Set $\delta_{x}=x \partial_{x}$ and $\delta_{y}=y \partial_{y}$. Notice that

$$
\delta_{x} \varphi\left(\frac{y^{k}}{x^{n}}\right)=-n\left(\delta_{z} \varphi\right)\left(\frac{y^{k}}{x^{n}}\right), \quad \delta_{y} \varphi\left(\frac{y^{k}}{x^{n}}\right)=k\left(\delta_{z} \varphi\right)\left(\frac{y^{k}}{x^{n}}\right) .
$$

Since $\delta_{x} u_{i}=-(n / k) x^{\alpha_{i}+1} \partial_{y} u_{i+1}$,

$$
\left[\frac{y^{k}}{x^{n}} \prod_{j=0}^{k-2}\left(\delta_{x}-\sum_{i=0}^{j} \alpha_{i}-j-1\right) \delta_{x}-\left(-\frac{n}{k}\right)^{k} y^{k} \partial_{y}^{k}\right] u=0
$$

hence,

$$
\left[\prod_{j=0}^{k-1}\left(\delta_{z}-\frac{j}{k}+\frac{\lambda}{n}\right)-z \delta_{z} \prod_{j=0}^{k-2}\left(\delta_{z}+\frac{1}{n} \sum_{i=0}^{j}\left(\alpha_{i}+j+1\right)\right)\right] \varphi=0 .
$$

Therefore $D(\widetilde{\alpha}, \widetilde{\beta}) \varphi=0$.

\section{References}

[1] F. Beukers and G. Heckman, The monodromy of the hypergeometric function ${ }_{n} F_{n-1}$, Invent. Math., 95 (1989), 325-354, MR 90f:11034, Zbl 0663.30044.

[2] J.-E. Bjork, Analytic D-modules and Applications, Kluwer, 1993, MR 95f:32014, Zbl 0805.32001.

[3] C. Delorme, Sur les modules des singularités des courbes planes, Bull. Soc. Math. France, 106 (1978), 417-446, MR 80c:14019, Zbl 0395.14010. 
[4] G.-M. Greuel and G. Pfister, Moduli spaces for torsion free modules on curve singularities I, J. Alg. Geom., 2 (1993), 81-135, MR 93m:14006, Zbl 0785.14016.

[5] M. Kashiwara, Systems of Microdifferential Equations (Notes by T.M. Fernandes), Birkhäuser, 1983, MR 86b:58113, Zbl 0521.58057.

[6] _ Introduction to Microlocal Analysis, Monographie, 32, de l'Enseignement Mathematique, 1986, MR 88a:58187, Zbl 0592.58051.

[7] M. Kashiwara and T. Kawai, On holonomic systems of microdifferential equations III, Publ. RIMS, Kyoto Univ., 17 (1981), 813-979, MR 83e:58085, Zbl 0505.58033.

[8] _ Microlocal analysis, in 'Publ. RIMS', Kyoto Univ., 19 (1983), 1003-1032, MR 85h:58159, Zbl 0536.58030.

[9] N.M. Katz, Rigid Local Systems, Princ. Univ. Press, Princeton, 1996, MR 97e:14027, Zbl 0864.14013.

[10] M. Kohno, Global Analysis in Linear Differential Equations, Kluwer Academic Publishers, 1999, MR 2000h:34150, Zbl 0933.34002.

[11] A.H. Levelt, Hypergeometric functions, Indag. Math., 23 (1961), 361-403, MR 25 \#1301, Zbl 0124.03602.

[12] R. MacPherson and K. Vilonen, Elementary construction of perverse sheaves, Invent. Math., 84 (1986), 403-435, MR 87m:32028, Zbl 0597.18005.

[13] Ph. Maisonobe, Faisceaux pervers dont le suport singulier est une courbe plane, Comp. Math., 62 (1987), 215-261, MR 89c:32024, Zbl 0641.32008.

[14] L. Narváez-Macarro, Cycles évanescents et faisceaux pervers: Cas des courbes planes irreducibles, Comp. Math., 65 (1988), 321-347, MR 89k:32022, Zbl 0651.14013.

[15] O. Neto, A microlocal Riemann-Hilbert correspondence, Comp. Math, 127 (2001), 229-241, CMP 1845036.

[16] T. Oshima, On the Theorem of Cauchy-Kowalesky for first order linear differential equations, Proc. Japan Acad., 49 (1973), 83-87, MR 48 \#4458, Zbl 0283.35002.

[17] _ Singularities in contact geometry and degenerate pseudo-differential equations, J. Fac. Sci. Univ. Tokyo Sec. IA, 21 (1974), 43-83, MR 50 \#5862, Zbl 0282.35070.

[18] M. Sato, M. Kashiwara, T. Kimura and T. Oshima, Micro-local analysis of prehomogeneous vector spaces, Inventiones Math., 62 (1980), 117-178, MR 83g:32016, Zbl 0456.58034.

[19] M. Sato, T. Kawai and M. Kashiwara, Hyperfunctions and Pseudo-differential Equations, Lecture Notes in Mathematics, 287, Springer-Verlag, 1973, MR 57 \#17726, Zbl 0277.46039.

[20] P. Schapira, Microdifferential Systems in the Complex Domain, Springer-Verlag, 1985, MR 87k:58251, Zbl 0554.32022.

[21] P.C. Silva, Estrutura Local dos Sistemas Holónomos Regulares, Ph.D. Thesis, Universidade de Lisboa, (in preparation).

[22] C.T. Simpson, Products of Matrices, in 'Canadian Math. Soc. Conference Proc.', 12 (1992), 157-185, MR 93c:15015, Zbl 0756.15022.

[23] J. Thomae, Über die höheren hypergeomethrischen, Reihen. Math. Ann., 2 (1870), 427-444. 
[24] W. Wasow, Asymptotic Expansions for Ordinary Differential Equations, Interscience, New York, 1965, MR 34 \#3041, Zbl 0133.35301.

Received November 6, 1998 and revised May 24, 2002. This paper was partially supported by project PBICMAT215395 and TMR project ERBFMRXCT960040. The second named author was partially supported by JNICT's scholarship CIENCIA/BD284193RM.

CMAF, Universidade de Lisboa

Av. Gama Pinto, 2

1649-003 Lisboa Portugal

E-mail address: orlando@lmc.fc.ul.pt

CMAF, Universidade DE Lisboa

Av. Gama Pinto, 2

1649-003 Lisboa Portugal

E-mail address: pcsilva@lmc.fc.ul.pt 